\title{
Versões não-lineares e vetoriais do Teorema de Banach-Stone
}

\author{
André Luis Porto da Silva
}

TESE APRESENTADA

$\mathrm{AO}$

Instituto de MatemáticA e EstatísticA

DA

Universidade DE SÃo PAUlo

PARA

OBTENÇÃO DO TÍTULO

$\mathrm{DE}$

DOUTOR EM CIÊNCIAS

Programa: Matemática

Orientador: Prof. Dr. Elói Medina Galego

Durante o desenvolvimento deste trabalho o autor recebeu auxílio financeiro da CAPES e do $\mathrm{CNPq}$ 


\title{
Versões não-lineares e vetoriais do Teorema de Banach-Stone
}

\begin{abstract}
Esta versão da tese contém as correções e alterações sugeridas pela Comissão Julgadora durante a defesa da versão original do trabalho, realizada em 20/09/2019. Uma cópia da versão original está disponível no Instituto de Matemática e Estatística da Universidade de São Paulo.
\end{abstract}

Comissão Julgadora:

- Prof. Dr. Eloi Medina Galego (orientador) - IME-USP

- Prof. Dr. Ricardo Bianconi - IME-USP

- Prof. Dr. Leandro Fiorini Aurichi - ICMC-USP

- Prof. Dr. Antonio Roberto da Silva - UFRJ

- Prof. Dr. Vinicius Cifú Lopes - UFABC 


\section{Agradecimentos}

Ao longo dos quatro anos de desenvolvimento desse trabalho, muitas foram as pessoas que contribuíram para que esse chegasse à sua conclusão.

Eu dedico este trabalho, inteiramente, à minha esposa e companheira de todos os dias, Letícia, por ter me apoiado desde os momentos de verborragia eufórica, que terminavam em rabiscos e garranchos de ideias matemáticas para as quais teve a paciência de acenar e acolher minha paixão por elas; até os momentos do pesar da ansiedade e insegurança, inerentes ao fim de toda tese de doutorado, os quais só pude superar com o seu apoio.

Agradeço imensamente à minha família, por todo o suporte que tive desde minha infância, quando perdi meus pais, até a fase adulta, quando vez ou outra demando um cuidado especial de casa. Em especial, agradeço à minha irmã que morou comigo nos últimos anos e com a qual pude compartilhar um cotidiano intenso e vivo, regado a café, incenso, cerveja, extensas conversas e sempre uma nova ideia.

Cabe um agradecimento especial aos meus sogros, Luciano e Francisca, e aos meus cunhados, Mariana e Henrique, por terem me acolhido como a um membro da família no momento mais delicado da minha vida: quando passei pelo tratamento de um câncer no pericárdio. Durante os oito meses de tratamento me receberam em casa e me deram todo o auxílio e companhia de que necessitei. Não importa o que eu faça em retorno, sempre me considerarei em débito com vocês por tudo o que fizeram e fazem por mim.

Aos meus companheiros de sala: Hugo, Felipe, Yuri, João, Rodrigo, André Zaidan e Gilson. Foi com eles que pude compartilhar a rotina universitária de meus últimos anos de doutorado. É sempre muito bom entrar na sala B-141 e encontrá-los para compartilhar um café. Sou muito grato pelas conversas (intermináveis), o companheirismo e inspiração.

Agradeço ao meu orientador Eloi Medina Galego, com o qual sempre pude contar com as novas ideias para problemas, as discussões e a paciência. Tenho muito orgulho e gratidão pelo nosso frutuoso trabalho juntos.

Agradeço a CAPES e a CNPq pelo financiamento de minha pesquisa por meio do qual fora possível minha dedicação exclusiva a este trabalho.

Por fim, agradeço aos professores Ricardo Bianconi, Leandro Fiorini Aurichi, Antonio Roberto da Silva e Vinicius Cifú Lopes por terem aceitado fazer parte da minha banca de defesa. 



\section{Resumo}

SILVA, A. L. P. Versões não-lineares e vetoriais do Teorema de Banach-Stone. 2019. 44 p. Tese (Doutorado) - Instituto de Matemática e Estatística, Universidade de São Paulo, São Paulo, 2019.

Seja $X$ um espaço de Banach de dimensão finita e $K, S$ espaços de Hausdorff localmente compactos. Nessa tese de doutorado, lidamos com o problema de quando uma função $T$ de $C_{0}(K, X)$ sobre $C_{0}(S, X)$ implica que $K$ e $S$ são homeomorfos. Para esse propósito, apresentamos uma nova técnica, inspirada na prova de um resultado clássico de Jarosz (1989), que nos dá versões do teorema de Banach-Stone para funções bijetoras $T: C_{0}(K, X) \rightarrow C_{0}(S, X)$ satisfazendo

$$
\frac{1}{M}\|f-g\|-L \leq\|T(f)-T(g)\| \leq M\|f-g\|+L,
$$

para toda $f, g \in C_{0}(K, X)$. Esse é o resultado de um projeto de longa data, desde o trabalho de mestrado do autor, e envolveu um extenso estudo de artigos escritos por Cambern, Jarosz, Dutriex, Kalton, Górak, entre outros. No que segue, formalizamos essa técnica, depois discutimos os resultados provenientes dela e apresentamos as provas detalhadas dos dois teoremas mais importantes. $\mathrm{O}$ primeiro teorema garante que $K$ e $S$ são homeomorfos sempre que $L \geq 0$ e $1 \leq M^{2}<\mathcal{S}(X)$, onde $\mathcal{S}(X)$ denota a constante de Schäffer de $X$, estendendo e unificando alguns resultados lineares e vetoriais para o contexto não-linear. O segundo teorema nos dá uma extensão da versão clássica do teorema de Banach-Stone para espaços de Hilbert, provada por Cambern, para isomorfismos com distorção maior que $\sqrt{2}$, resolvendo um antigo problema em aberto.

Palavras-chave: teorema de Banach-Stone, teorema de Amir-Cambern, quasi-isometrias, constante de Schäffer. 



\section{Abstract}

SILVA, A. L. P. Nonlinear and vector versions of the Banach-Stone theorem. 2019. 44 p. Tese (Doutorado) - Instituto de Matemática e Estatística, Universidade de São Paulo, São Paulo, 2019.

Let $X$ be a finite-dimensional Banach space and $K, S$ be locally compact Hausdorff spaces. In this doctoral thesis, we deal with the problem of whether a map $T$ from $C_{0}(K, X)$ onto $C_{0}(S, X)$ implies that $K$ and $S$ are homeomorphic. For that purpose, we present a brand new technique, inspired by the proof of a classical result by Jarosz (1989), which gives us versions of the BanachStone theorem for bijective maps $T: C_{0}(K, X) \rightarrow C_{0}(S, X)$ satisfying

$$
\frac{1}{M}\|f-g\|-L \leq\|T(f)-T(g)\| \leq M\|f-g\|+L
$$

for every $f, g \in C_{0}(K, X)$. This is the result of a longstanding project, since the author's masters work, and involved the extensive study of several papers writen by Cambern, Jarosz, Dutriex, Kalton, Górak, among others. In what follows, we formalize this technique, then we discuss the results provided by it and present detailed proofs of the two most important theorems. The first one states that $K$ and $S$ are homeomorphic whenever $L \geq 0$ and $1 \leq M^{2}<\mathcal{S}(X)$, where $\mathcal{S}(X)$ denotes the Schäffer constant of $X$, extending and unifying several linear versions to a nonlinear context. The second one provides an extension of the classical linear version of Banach-Stone theorem for Hilbert spaces, proved by Cambern, to isomorphisms with distortion greater than $\sqrt{2}$, solving a longstanding open problem.

Keywords: Banach-Stone theorem, Amir-Cambern theorem, quasi-isometries, Schäffer's constant. 



\section{Sumário}

1 Introdução $\quad 1$

1.1 Versões Não-lineares Reais . . . . . . . . . . . . . . . . . . . . . . . . . 2

1.2 Versões Não-lineares Vetoriais . . . . . . . . . . . . . . . . . . . . . . . . 4

1.3 Versões Lineares Vetoriais . . . . . . . . . . . . . . . . . . . . . . . 5

2 Preliminares $\quad 9$

2.1 Redes . . . . . . . . . . . . . . . . . . . . . . . . . 9

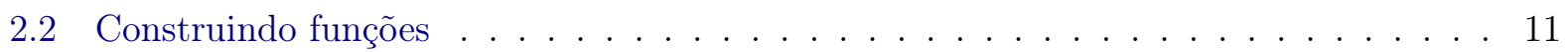

2.3 Sobre a constante de Schäffer . . . . . . . . . . . . . . . . . . . . . . 12

3 Os conjuntos de aproximação $\quad 15$

3.1 Definição dos conjuntos de aproximação . . . . . . . . . . . . . . . . . . . 15

3.2 Lema da existência . . . . . . . . . . . . . . . . . . . . . . 17

3.3 Lema da simetria . . . . . . . . . . . . . . . . . . . . . . . . 21

3.4 A propriedade fundamental dos conjuntos de aproximação . . . . . . . . . . . . 22

4 Versão não-linear e vetorial para o Teorema de Amir-Cambern 25

5 Versão linear vetorial $\quad 29$

5.1 Sobre a existência de constantes de Cambern . . . . . . . . . . . . . . . . . 31

5.2 Uma versão linear e vetorial para constantes de Cambern . . . . . . . . . . . . . . 34

6 Conclusões $\quad 41$

6.1 Considerações Finais . . . . . . . . . . . . . . . . . . . . . . . 41

6.2 Sugestões para Pesquisas Futuras . . . . . . . . . . . . . . . . . . . . . . 41

Referências Bibliográficas $\quad 43$ 



\section{Capítulo 1}

\section{Introdução}

Seja $K$ um espaço de Hausdorff localmente compacto e $X$ um espaço de Banach. Denotaremos por $C_{0}(K, X)$ o espaço de Banach das funções contínuas de $K$ a valores em $X$ que se anulam no infinito, equipado com a norma do supremo. Quando $K$ for compacto, o espaço $C_{0}(K, X)$ será denotado por $C(K, X)$. Denotaremos $C_{0}(K, \mathbb{R})$ e $C(K, \mathbb{R})$ por $C_{0}(K)$ e $C(K)$, respectivamente.

No que segue, sempre estaremos considerando a estrutura de espaço de Banach de $C_{0}(K, X)$, portanto, ao longo de todo o texto, quando fizermos uso do termo isomorfismo, estaremos nos referindo a isomorfismos lineares.

A motivação central do nosso trabalho é o Teorema de Banach-Stone, que estabelece que a estrutura métrica de $C_{0}(K)$ determina a topologia de $K$ [Beh78, p. 138]. Mais precisamente:

Teorema 1.1. Sejam $K$ e $S$ espaços de Hausdorff localmente compactos e $T$ um isomorfismo isométrico de $C_{0}(K)$ sobre $C_{0}(S)$. Então:

(a) K e $S$ são homeomorfos;

(b) existe um homeomorfismo $\phi: S \rightarrow K$ e uma função contínua $\lambda: S \rightarrow\{-1,1\}$ tal que para todo $s \in S$ e $f \in C_{0}(K)$, temos

$$
T f(s)=\lambda(s) f(\phi(s))
$$

A primeira versão desse teorema foi provada em 1933, por Banach [Ban33], para $K$ e $S$ compactos metrizáveis. Em 1937, Stone [Sto37] estendeu o resultado para espaços de Hausdorff compactos. Desde então, houve uma grande quantidade de extensões, generalizações e variantes do teorema em diferentes contextos [CGRV15, e suas referências]. Destacaremos abaixo algumas versões do teorema que serão pontos fundamentais para o nosso estudo.

O Teorema de Amir-Cambern, provado de modo independente por Amir [Ami65], em 1965, e Cambern [Cam66] [Cam67], em 1967, foi a primeira generalização do Teorema de Banach-Stone que enfraqueceu a hipótese sobre a isometria entre os espaços $C_{0}(K)$ e $C_{0}(S)$.

Teorema 1.2. Sejam K e $S$ espaços de Hausdorff localmente compactos. Se existe um isomorfismo $T$ de $C_{0}(K)$ sobre $C_{0}(S)$ tal que $\|T\|\|T\|^{-1}<2$, então $K$ e $S$ são homeomorfos.

Em 1975, Cohen [Coh75] obteve um contra-exemplo, mostrando que não é possível aumentar a constante 2 na formulação do teorema acima, mesmo assumindo que $K$ e $S$ sejam compactos. Mais precisamente, o autor exibiu compactos não-homeomorfos $K$ e $S$ e um isomorfismo $T$ de $C(K)$ sobre $C(S)$ satisfazendo $\|T\|\|T\|^{-1}=2$.

Muitos autores, começando com Jerison [Jer50], consideraram o problema de determinar propriedades geométricas de $X$ que permitissem generalizações desse teorema para os espaços $C_{0}(K, X)$. Em 1976, Cambern [Cam76] fez a primeira tentativa de obter uma versão do Teorema de BanachStone para espaços $C_{0}(K, X)$, com $\operatorname{dim} X>1$, via isomorfismos $T$ com $\|T\|\left\|T^{-1}\right\|>1$, em que então provou: 
Teorema 1.3. Sejam K e $S$ espaços de Hausdorff localmente compactos e $X$ um espaço de Hilbert de dimensão finita. Se existe um isomorfismo $T$ de $C_{0}(K, X)$ sobre $C_{0}(S, X)$ tal que $\|T\|\left\|T^{-1}\right\|<\sqrt{2}$, então $K$ e $S$ são homeomorfos.

Tendo em vista o contra-exemplo de Cohen, que atesta a otimalidade do Teorema 1.2, surge a questão se $\sqrt{2}$ é o maior número possível para a formulação do Teorema 1.3, que permanece em aberto.

Em 1989, dois resultados obtidos por Jarosz (Teoremas 1.6 e 1.12), mostraram ser possível enfraquecer não só a hipótese sobre a isometria, como também sobre a linearidade de $T$, dando início à pesquisa de versões do Teorema de Banach-Stone via funções $T$ não-necessariamente lineares.

No que segue, as versões do Teorema de Banach-Stone que abrangem espaços $C_{0}(K, X)$, com $\operatorname{dim} X>1$, são chamadas de versões vetoriais, e as que consideram apenas espaços $C_{0}(K)$ são chamadas de versões reais. Além disso, as versões que abrangem somente funções lineares são chamadas de versões lineares, e as que abrangem funções não-necessariamente lineares são chamadas de versões não-lineares.

Na presente tese, temos como objetivo geral investigar, à luz de resultados precedentes, novas versões do Teorema de Banach-Stone em três frentes: versões não-lineares reais, versões não-lineares vetoriais e versões lineares vetoriais. Nas seções seguintes, discorremos sobre os objetivos específicos, bem como os resultados alcançados de cada uma das frentes.

\subsection{Versões Não-lineares Reais}

A seguir apresentamos um histórico de resultados não-lineares através de uma classe de funções não-necessariamente lineares, as quasi-isometrias, introduzida em [Bri08].

Definição 1.4. Sejam $E$ e $F$ espaços de Banach. Dizemos que $T: E \rightarrow F$ é uma $(M, L)$-quasiisometria (ou apenas quasi-isometria) se

- $T$ satisfaz

$$
\frac{1}{M}\|x-y\|-L \leq\|T(x)-T(y)\| \leq M\|x-y\|+L, \quad \forall x, y \in E ;
$$

- ImT é $\xi$-densa em $F$ (isto é, $\forall u \in F \exists x:\|u-T(x)\|<\xi$ ), para algum $\xi>0$.

As quasi-isometrias englobam conceitos importantes utilizados na classificação não-linear de espaços de Banach, por exemplo, distância net, funções Lipschitz para grandes distâncias e homeomorfismos uniformes (para mais detalhes, veja [BL00, GLZ14, Gór11a, Kal08]).

A classe das quasi-isometrias será discutida com mais detalhes do capítulo 3 em diante. Destacamos abaixo uma importante conexão entre os conceitos de isomorfismo e quasi-isometria:

Observação 1.5. Para todo isomorfismo T, o isomorfismo definido por

$$
\tau=\sqrt{\frac{\left\|T^{-1}\right\|}{\|T\|}} T,
$$

é uma $\left(\sqrt{\|T\|\left\|T^{-1}\right\|}, 0\right)$-quasi-isometria bijetora.

Em [Jar89, Theorem 1], Jarosz provou a primeira versão não-linear real do Teorema de BanachStone, mostrando que a linearidade não é necessária se $T$ for uma função suficientemente próxima de ser uma isometria. Enunciamos este resultado abaixo.

Teorema 1.6. Existe $\varepsilon_{0}>0$ tal que, para quaisquer espaços de Hausdorff localmente compactos $K$ e $S$, a existência de uma $(M, 0)$-quasi-isometria bijetora de $C_{0}(K)$ sobre $C_{0}(S)$ com $M<1+\varepsilon_{0}$ implica que $K$ e $S$ são homeomorfos. 
A demonstração do teorema acima nos garante apenas valores para $\varepsilon_{0}$ menores que $10^{-30}$. Em 2005, Dutriex \& Kalton [DK05] obtiveram uma melhora significativa da restrição sobre $M$, provando que o Teorema 1.6 continua válido se $T$ for uma $(M, L)$-quasi-isometria bijetora com $M<\sqrt{17 / 16}$. Posteriormente, em 2011, dois teoremas provados por Górak [Gór11a] [Gór11b], aumentaram a constante $\sqrt{17 / 16}$ para $\sqrt{6 / 5}$, no caso em que $K$ e $S$ são assumidos compactos; e para $\sqrt{16 / 15}$, no caso geral. São eles:

Teorema 1.7. Sejam $K$ e $S$ espaços de Hausdorff compactos. Seja $T: C(K) \rightarrow C(S)$ uma $(M, L)$ quasi-isometria bijetora tal que $T(0)=0$. Então, para todo $M<\sqrt{6 / 5}$ existe um homeomorfismo $\varphi: S \rightarrow K$ e uma função contínua $\lambda: S \rightarrow\{-1,1\}$ tal que, para todo $s \in S$ e $f \in C(K)$,

$$
|T f(s)-\lambda(s) f(\varphi(s))| \leq 26(M-1)\|f\|+\Delta,
$$

onde $\Delta$ não depende de $f$ e $s$, e $\Delta=0$ quando $L=0$.

Teorema 1.8. Sejam $K$ e $S$ espaços de Hausdorff localmente compactos. Seja $T: C_{0}(K) \rightarrow C_{0}(S)$ uma $(M, L)$-quasi-isometria bijetora tal que $T(0)=0$. Então, para todo $M<\sqrt{16 / 15}$ existe um homeomorfismo $\varphi: S \rightarrow K$ e uma função contínua $\lambda: S \rightarrow\{-1,1\}$ tal que para todo $s \in S e$ $f \in C_{0}(K)$,

$$
|T f(s)-\lambda(s) f(\varphi(s))| \leq 76(M-1)\|f\|+\Delta,
$$

onde $\Delta$ não depende de $f$ e $s$, e $\Delta=0$ quando $L=0$.

Pela Observação 1.5 e o contra-exemplo apresentado por Cohen (veja página 1), temos que a maior constante possível na formulação dos Teoremas 1.7 e 1.8 é $\sqrt{2}$. Com isso em vista, em [Gór11a], Górak propôs os seguintes problemas:

Problema 1.9. É verdade que, para todos os espaços de Hausdorff compactos $K$ e $S$, a existência de uma $(M, L)$-quasi-isometria bijetora de $C(K)$ sobre $C(S)$ com $M<\sqrt{2}$ implica que $K$ e $S$ são homeomorfos?

Problema 1.10. Qual a resposta para o problema 1.9 se supusermos que $K$ e $S$ são compactos enumeráveis? E se $K=\left\{\frac{1}{n}: n \in \mathbb{N}\right\} \cup\{0\}$ ?

Aplicando uma modificação das técnicas utilizadas por Górak na demonstração do Teorema 1.7, obtivemos uma resposta afirmativa para o Problema 1.9, inclusive no caso geral em que se requer apenas que os espaços $K$ e $S$ sejam localmente compactos. Mais precisamente, provamos em [GPdS16], o seguinte teorema.

Teorema 1.11. Sejam $K$ e $S$ espaços de Hausdorff localmente compactos. Seja $T: C_{0}(K) \rightarrow C_{0}(S)$ uma $(M, L)$-quasi-isometria bijetora com $M<\sqrt{2}$. Então:

(a) K e $S$ são homeomorfos;

(b) existe um homeomorfismo $\varphi: S \rightarrow K$ e uma função contínua $\lambda: S \rightarrow\{-1,1\}$ tal que para todo $s \in S$ e $f \in C_{0}(K)$ temos

$$
|M T f(s)-\lambda(s) f(\varphi(s))| \leq\left(M^{2}-1\right)\|f\|+\Delta,
$$

onde $\Delta$ não depende de $f$ e $s$ e $\Delta=0$ quando $L=0$.

Como vimos, o teorema acima é ótimo, no sentido que a constante $\sqrt{2}$ é a maior possível na sua formulação. Note que, pela Observação 1.5, o Teorema 1.11 também generaliza o Teorema 1.2, de Amir-Cambern.

Além disso, a estimativa da distância de $T$ a uma isometria, dada pelo Teorema 1.11.(b), melhora as estimativas dadas por Amir e Cambern. Segue de suas demonstrações que se $U: C_{0}(K) \rightarrow C_{0}(S)$ é um isomorfismo sobrejetor e $\varepsilon<1$ satisfaz

$$
\|f\| \leq\|U(f)\| \leq(1+\varepsilon)\|f\|,
$$


para toda $f \in C_{0}(K)$, então existe um isomorfismo isométrico $V: C_{0}(K) \rightarrow C_{0}(S)$ satisfazendo $\|U-V\|<3 \varepsilon$ (veja [Ves04, p. 204]). Nossa estimativa permite reduzir $3 \varepsilon$ para $\varepsilon$, o menor número possível. Para ver isso, faça $M=\sqrt{1+\varepsilon}$ e note que o isomorfismo $T=U / M$ é uma $(M, 0)$-quasiisometria bijetora. Então, pelo Teorema 1.11, existe uma isometria $V: C_{0}(K) \rightarrow C_{0}(S)$, definida por $V(f)(s)=\lambda(s) f(\varphi(s))$, tal que

$$
|U(f)(s)-V(f)(s)|=|M T f(s)-\lambda(s) f(\varphi(s))| \leq\left(M^{2}-1\right)\|f\|=\varepsilon\|f\| .
$$

Para ver que $\varepsilon$ é o menor número para esse tipo de estimativa, basta considerar $U: C_{0}(K) \rightarrow C_{0}(K)$ dado por $U(f)=(1+\varepsilon) f$.

Por fim, observamos que a estimativa dada no item (b) do Teorema 1.11 melhora ambas as estimativas dadas pelos resultados de Górak (Teoremas 1.7 e 1.8). Para ver isso, note que, a partir da estimativa dada pelo item (b) do Teorema 1.11, derivamos o seguinte:

$$
\begin{aligned}
|T f(s)-\lambda(s) f(\varphi(s))| & \leq M^{-1}|M T f(s)-\lambda(s) f(\varphi(s))|+\left(1-M^{-1}\right)|\lambda(s) f(\varphi(s))| \\
& \leq M^{-1}\left(\left(M^{2}-1\right)\|f\|+\Delta\right)+\left(1-M^{-1}\right)\|f\| \\
& \leq\left(M+1-2 M^{-1}\right)\|f\|+\frac{\Delta}{M}
\end{aligned}
$$

e então

$$
M+1-2 M^{-1}=\left(1+2 M^{-1}\right)(M-1)<26(M-1),
$$

para todo $1<M<\sqrt{2}$.

\subsection{Versões Não-lineares Vetoriais}

Também decorre do trabalho de Jarosz a primeira versão não-linear vetorial do Teorema de Banach-Stone [Jar89, Theorem 4], enunciada abaixo.

Teorema 1.12. Seja $X$ um espaço de Banach estritamente convexo de dimensão finita. Existe $\varepsilon_{0}>0$, dependendo de $X$, tal que, para quaisquer espaços de Hausdorff localmente compactos $K$ e $S$, a existência de uma $(M, 0)$-quasi-isometria bijetora de $C_{0}(K, X)$ sobre $C_{0}(S, X)$ com $M<1+\varepsilon_{0}$ implica que $K$ e $S$ são homeomorfos.

Mesmo no caso em que $X=\mathbb{R}$, os argumentos apresentados na prova de [Jar89, Theorem 1] requerem que $\epsilon_{0}$ seja extremamente pequeno, mais precisamente $\epsilon_{0}<10^{-30}$. Além disso, se $X$ tem dimensão maior ou igual que $2, \epsilon_{0}$ depende do módulo de convexidade de $X$ e nada é estabelecido sobre ele além de sua existência.

Provada a possibilidade de se obter versões não-lineares vetoriais para o Teorema 1.1 para o caso em que $X$ é estritamente convexo e de dimensão finita, surge naturalmente o problema de estabelecer valores maiores para $\varepsilon_{0}$.

A eficácia das técnicas obtidas durante a demonstração do Teorema 1.11, nos sugeriu a aplicação destas, transpostas para o contexto vetorial, no intuito de melhorar o Teorema 1.12. Entretanto, a aplicação de tais técnicas depende fortemente das propriedades dos espaços de Banach $X$ que consideramos. Num primeiro momento, abordamos os espaços de Hilbert de dimensão finita. Mais precisamente, atacamos o seguinte problema:

Problema 1.13. Quando uma quasi-isometria de espaços $C_{0}(K, X)$ determina a topologia dos espaços localmente compactos $K$ para todo espaço de Hilbert $X$ de dimensão finita maior ou igual que 2 ?

Como resposta ao problema acima, a partir de alterações da técnica aplicada na demonstração do Teorema 1.11 (uma versão preliminar da técnica dos conjuntos de aproximação), provamos, em [GPdS18b], o seguinte teorema: 
Teorema 1.14. Sejam K e $S$ espaços de Hausdorff localmente compactos e $X$ um espaço de Hilbert de dimensão finita maior ou igual a 2. Se existe uma $(M, L)$-quasi isometria $T$ de $C_{0}(K, X)$ sobre $C_{0}(S, X)$ com $M<\sqrt[4]{2}$, então $K$ e $S$ são homeomorfos.

Note que, pela Observação 1.5, o teorema acima generaliza o Teorema 1.3, alcançando assim o melhor resultado para espaços de Hilbert até então.

O próximo passo, nessa linha, foi investigar resultados análogos ao Teorema 1.14 para uma classe maior de espaços de Banach. Como inspiração, temos o seguinte resultado, provado em [CGRV15].

Teorema 1.15. Sejam K e $S$ espaços de Hausdorff localmente compactos e $X$ um espaço de Banach real. Se existe um isomorfismo $T$ de $C_{0}(K, X)$ sobre $C_{0}(S, X)$ satisfazendo $\|T\|\left\|T^{-1}\right\|<\mathcal{S}(X)$, então $K$ e $S$ são homeomorfos.

O parâmetro $\mathcal{S}(X)$ utilizado acima é a constante de Schäffer de $X$, definida por:

$$
\mathcal{S}(X)=\inf \{\max \{\|x+y\|,\|x-y\|\}:\|x\|=\|y\|=1\} .
$$

A constante $\mathcal{S}(X)$ será vista com mais detalhes nos capítulos 2 e 4. Por ora, convém destacar apenas que $\mathcal{S}(\mathbb{R})=2$ e que $\mathcal{S}(X)=\sqrt{2}$ quando $X$ é um espaço de Hilbert com dimensão maior ou igual que 2 (veja Proposição 2.16). Portanto, o Teorema 1.15 unifica os Teoremas 1.2 e 1.3.

Avançando no desenvolvimento da técnica dos conjuntos de aproximação, provamos, em [GPdS18a], o teorema abaixo, que constitui uma versão não-linear do Teorema 1.15.

Teorema 1.16. Sejam K e $S$ espaços de Hausdorff localmente compactos e $X$ um espaço de Banach de dimensão finita. Se existe uma $(M, L)$-quasi isometria $T$ de $C_{0}(K, X)$ sobre $C_{0}(S, X)$ com $M<$ $\sqrt{\mathcal{S}(X)}$. Então K e $S$ são homeomorfos.

Note que, pela Observação 1.5, esse Teorema generaliza o Teorema 1.15 para a classe das quasiisometrias, no caso em que $X$ é um espaço de Banach de dimensão finita. Sendo assim, mais uma vez, nossas técnicas nos deram um resultado não-linear que alcança o resultado linear que o motivou.

Por conta de $\mathcal{S}(\mathbb{R})=\sqrt{2}$ e $\mathcal{S}(X)=\sqrt{2}$ para todo $X$ Hilbert de dimensão maior ou igual que 2 , o Teorema 1.16 unifica os Teoremas 1.11 e 1.14 .

No caso especial dos espaços $X=\ell_{p}^{n}$, o espaço $\ell_{p} n$-dimensional, com $1<p<\infty$ e $n \geq 2$, o seguinte corolário do Teorema 1.16 só era conhecido quando $p=2$.

Corolário 1.17. Sejam $1<p<\infty$ e $n \geq 2$. Suponha que $K$ e $S$ são espaços de Hausdorff localmente compactos e $T$ é uma $(M, L)$-quasi-isometria bijetora de $C_{0}\left(K, \ell_{p}^{n}\right)$ sobre $C_{0}\left(S, \ell_{p}^{n}\right)$, satisfazendo

$$
M^{2}<\min \left\{2^{1 / p}, 2^{1-1 / p}\right\}
$$

então, $K$ e $S$ são homeomorfos.

Demonstração. Pelo item (3) da Proposição 2.16, para quaisquer $1<p<\infty$ e $n \geq 2$, temos

$$
\mathcal{S}\left(\ell_{p}^{n}\right)=\min \left\{2^{1 / p}, 2^{1-1 / p}\right\}
$$

A partir disso, basta aplicar o Teorema 1.16.

Por outro lado, Teorema 1.16 não se aplica para $X=\ell_{\infty}^{n}, n \geq 2$, o espaço $\ell_{\infty} n$-dimensional, porque, nesse caso, $\mathcal{S}(X)=1$ e, além disso, por um resultado de Sundaresan [Sun73, p.22], existem espaços de Hausdorff compactos $K$ e $S$ não-homeomorfos tais que $C\left(K, \ell_{\infty}^{n}\right)$ é isométrico a $C\left(S, \ell_{\infty}^{n}\right)$.

\subsection{Versões Lineares Vetoriais}

Em nosso estudo de versões lineares vetoriais, inspirados pelo Teorema 1.3, consideramos principalmente o seguinte problema, proposto por Cambern em [Cam76]: 
Problema 1.18. É possível estender o Teorema 1.3 para $\|T\|\left\|T^{-1}\right\|<\delta$ para algum $\delta>\sqrt{2}$ ?

Esse problema é relevante não só por conta do Teorema 1.3, mas também pelo fato de que todas as versões lineares vetoriais conhecidas até então contemplavam apenas isomorfismos $T$ satisfazendo $1 \leq\|T\|\left\|T^{-1}\right\|<\sqrt{2}$.

É válido mencionar que o Teorema 1.3 não pode ser estendido para espaços de Hilbert de dimensão infinita, como ilustramos no exemplo a seguir:

Exemplo 1.19. De fato, seja $I$ um conjunto infinito e escreva $I=I_{1} \cup I_{2}$ tal que $I_{1} \cap I_{2}=\emptyset$ e as cardinalidades de $I_{1}$ e $I_{2}$ são iguais à cardinalidade de $I$. Sejam $K_{1}=\{1\}$ e $K_{2}=\{1,2\}$ compactos Hausdorff discretos. Considere as isometrias naturais

$$
\Theta: C\left(K_{2}, \ell_{2}(I)\right) \rightarrow \ell_{2}(I) \oplus_{\infty} \ell_{2}(I) \equiv \ell_{2}\left(I_{1}\right) \oplus_{\infty} \ell_{2}\left(I_{2}\right) \text { e } \Upsilon: \ell_{2}(I) \rightarrow C\left(K_{1}, \ell_{2}(I)\right)
$$

onde ' $\oplus_{\infty}$ ' denota o espaço normado da soma direta, munido da norma $\|(\mathrm{u}, \mathrm{v})\|=\max \{\|\mathrm{u}\|,\|\mathrm{v}\|\}$. Agora, defina $T: \ell_{2}\left(I_{1}\right) \oplus_{\infty} \ell_{2}\left(I_{2}\right) \rightarrow \ell_{2}(I)$ por

$$
T\left(\left(a_{i}\right)_{i \in I_{1}},\left(b_{i}\right)_{i \in I_{2}}\right)=\left(c_{i}\right)_{i \in I}
$$

onde $c_{i}=a_{i}$ se $i \in I_{1}$ e $c_{i}=b_{i}$ se $i \in I_{2}$. Então, é simples verificar que

$$
\|\Upsilon T \Theta\|=\sqrt{2} \text { e }\left\|(\Upsilon T \Theta)^{-1}\right\|=1
$$

Mas, é claro que $K_{1}$ e $K_{2}$ não são homeomorfos.

Tendo em vista o Problema 1.18 acima, inicialmente, atacamos uma forma mais fraca deste, considerando isomorfismos $T$ satisfazendo $\|T\|\left\|T^{-1}\right\|=\sqrt{2}$. Mais precisamente, consideramos:

Problema 1.20. É possível estender o Teorema 1.3 para $T$ satisfazendo $\|T\|\left\|T^{-1}\right\|=\sqrt{2}$ ?

As técnicas obtidas para a demonstração do Teorema 1.14 apresentaram propriedades interessantes no caso em que $T$ é linear. Como consequência de tais ferramentas, com as modificações específicas para o caso, obtivemos, em [GPdS17], uma resposta afirmativa para o problema acima.

Teorema 1.21. Sejam K e $S$ espaços de Hausdorff localmente compactos e $X$ um espaço de Hilbert de dimensão finita maior ou igual que 2. Suponha que existe um isomorfismo $T$ de $C_{0}(K, X)$ sobre $C_{0}(S, X)$ satisfazendo

$$
\frac{\|f\|}{\sqrt[4]{2}} \leq\|T(f)\| \leq \sqrt[4]{2}\|f\|,
$$

para toda $f \in C_{0}(K, X)$. Então $K$ e $S$ são homeomorfos.

Note que, pela Observação 1.5, a solução para o Problema 1.20 é consequência do Teorema 1.21. Como havíamos observado, o teorema acima foi a primeira versão linear vetorial do Teorema de Banach-Stone a abranger isomorfismos $T$ satisfazendo $\|T\|\left\|T^{-1}\right\|=\sqrt{2}$.

Em seguida, tendo em vista o Teorema 1.15, o passo seguinte de nossa pesquisa foi tentar uma versão análoga ao Teorema 1.21 para uma classe maior de espaços de Banach por meio do parâmetro $\mathcal{S}(X)$. Mais precisamente, atacamos o seguinte problema:

Problema 1.22. Para quais espaços de Banach reais $X$ é possível estender o Teorema 1.15 para $T$ satisfazendo $\|T\|\left\|T^{-1}\right\|=\mathcal{S}(X)$ ?

Como resultado dessa investigação, provamos em [GPdS19a] o seguinte teorema, que nos fornece uma resposta positiva para o problema acima no caso em que $X$ é estritamente convexo.

Teorema 1.23. Sejam K e $S$ espaços de Hausdorff localmente compactos e X um espaço de Banach estritamente convexo de dimensão finita maior ou igual que 2. Suponha que existe um isomorfismo $T$ de $C_{0}(K, X)$ sobre $C_{0}(S, X)$ satisfazendo

$$
\frac{\|f\|}{\sqrt{\mathcal{S}(X)}} \leq\|T(f)\| \leq \sqrt{\mathcal{S}(X)}\|f\|,
$$


para toda $f \in C_{0}(K, X)$. Então $K$ e $S$ são homeomorfos.

Por fim, após extensivo estudo da aplicação das técnicas no contexto linear vetorial alcançamos as ferramentas necessárias para atacar o Problema 1.18 considerado inicialmente, e obtivemos uma resposta afirmativa para ele. Mais precisamente, provamos em [GPdS19c] o seguinte:

Teorema 1.24. Seja $X$ um espaço de Hilbert real de dimensão $n \geq 2$. Existe $\delta_{n}>0$ tal que se $K$ e $S$ são espaços de Hausdorff localmente compactos e existe um isomorfismo $T$ de $C_{0}(K, X)$ sobre $C_{0}(S, X)$, satisfazendo

$$
\|T\|\left\|T^{-1}\right\|<\sqrt{2+\delta_{n}}
$$

para toda $f \in C_{0}(K, X)$, então $K$ e $S$ são homeomorfos.

Na verdade, o resultado acima também é válido para quando $X$ é um espaço de Hilbert complexo, como podemos ver na observação a seguir.

Observação 1.25. Todo espaço de Hilbert $X$ complexo de dimensão finita é isométrico ao espaço $\mathbb{C}_{2}^{n}$ das n-uplas de números complexos munido da 2-norma usual.

É simples verificar que $\left(z_{1}, \ldots, z_{n}\right) \mapsto\left(\operatorname{Re}\left(z_{1}\right), \operatorname{Im}\left(z_{1}\right), \operatorname{Re}\left(z_{2}\right), \operatorname{Im}\left(z_{2}\right), \ldots, \operatorname{Re}\left(z_{n}\right), \operatorname{Im}\left(z_{n}\right)\right)$ define uma isometria $\mathbb{R}$-linear de $\mathbb{C}_{2}^{n}$ sobre $\mathbb{R}_{2}^{2 n}$. Denotemos essa isometria por $I$. Por sua vez, I induz uma isometria $\mathbb{R}$-linear e sobrejetora $\mathcal{I}_{K}: C_{0}\left(K, \mathbb{C}_{2}^{n}\right) \rightarrow C_{0}\left(K, \mathbb{R}_{2}^{2 n}\right)$ dada por

$$
\mathcal{I}_{K}(f)(k)=I(f(k)), \forall k \in K .
$$

Portanto, a partir de um isomorfismo $\mathbb{C}$-linear $T: C_{0}\left(K, \mathbb{C}_{2}^{n}\right) \rightarrow C_{0}\left(S, \mathbb{C}_{2}^{n}\right)$, obtemos um isomorfismo $\mathbb{R}$-linear $\hat{T}=\mathcal{I}_{S} \circ T \circ \mathcal{I}_{K}^{-1}: C_{0}\left(K, \mathbb{R}_{2}^{2 n}\right) \rightarrow C_{0}\left(S, \mathbb{R}_{2}^{2 n}\right)$, satisfazendo $\|\hat{T}\|\left\|\hat{T}^{-1}\right\|=\|T\|\left\|T^{-1}\right\|$. Assim, para concluirmos que $K$ e $S$ são homeomorfos, basta aplicarmos o Teorema 1.24 para o isomorfismo $\hat{T}$.

Até então, o Teorema 1.24 é a única versão vetorial do Teorema de Banach-Stone que abrange isomorfismos $T$ com o valor de $\|T\|\left\|T^{-1}\right\|$ maior que $\sqrt{2}$. É importante destacar, no entanto, que o valor encontrado para $\delta_{n}$ ainda é baixo, mesmo para o caso em que $X$ tem dimensão 2 , para o qual obtemos $\delta_{2}<0,023$ (veja Lema 5.2), por conta disso uma possível direção futura na pesquisa seria continuar investigando a possibilidade de aumento de $\delta_{n}$.

As técnicas envolvidas nos novos resultados aqui citados (Teoremas 1.11, 1.14, 1.16, 1.21, 1.23 e 1.24) foram unificadas na técnica dos conjuntos de aproximação, a ser introduzida no capítulo 3.

É válido mencionar que, além dos teoremas acima, também provamos dois resultados oriundos de versões fracas da técnica dos conjuntos de aproximação. O primeiro, provado em [GPdS19b], é uma versão não-linear do Teorema de Cengiz [Cen73] (uma versão fraca do Teorema de BanachStone, envolvendo isomorfismos entre subespaços extremamente regulares de $C_{0}(K)$ e $C_{0}(S)$ ) que, como consequência, generaliza e unifica alguns resultados clássicos envolvendo os espaços $C_{0}(K)$ e $C_{0}^{(1)}(K)$. O segundo, provado em [GPdS20], corresponde a uma generalização não-linear para o Teorema de Holsztyński [Hol66] (uma versão fraca do Teorema de Banach-Stone, que abrange $T: C_{0}(K) \rightarrow C_{0}(S)$ isomorfismos com a imagem, não-necessariamente sobrejetores).

Alguns dos nossos resultados mencionados acima dependem de ajustes específicos na técnica. A fim de não comprometer a coesão do texto, optamos por expor apenas a demonstração dos Teoremas 1.16 e 1.24. Vale destacar novamente que o Teorema 1.16 generaliza os Teoremas 1.11 e 1.14, e o Teorema 1.24 generaliza o Teorema 1.21, portanto, a exposição a seguir contemplará a demonstração da maior parte dos resultados.

A seguir, temos o Capítulo 2, de preliminares, em que destacamos alguns conceitos fundamentais para o entendimento do trabalho. No Capítulo 3, introduzimos a técnica dos conjuntos de aproximação para espaços $C_{0}(K, X)$ com $X$ de dimensão finita e provamos que, mediante uma hipótese fundamental, obtemos um homeomorfismo entre $K$ e $S$. No capítulo 4, provamos o Teorema 1.16 e, no capítulo 5, o Teorema 1.24. Ambas as demonstrações serão decorrentes da técnica dos conjuntos de aproximação, mostrando, para cada caso, que a hipótese fundamental é satisfeita. Finalmente, no Capítulo 6, fazemos as considerações finais e apontamos direções futuras na pesquisa. 



\section{Capítulo 2}

\section{Preliminares}

Nas seções desse capítulo, introduzimos alguns conceitos fundamentais para o entendimento do trabalho. Começamos com um tratamento de redes em espaços topológicos, depois discutimos construções de funções em espaços $C_{0}(K, X)$ e por fim introduzimos algumas propriedades da constante de Schäffer.

\section{$2.1 \quad$ Redes}

A seguir é feita uma breve introdução de redes e estabelecidos resultados com respeito a lim sup e lim inf de redes em $\mathbb{R}$, dos quais faremos extensivo uso. O conteúdo a seguir refere-se à introdução feita por Megginson em [Meg12].

Definição 2.1 ([Meg12, p. 143]). Um conjunto dirigido é um conjunto não-vazio I com uma relação $\preceq$ em I tal que

(1) $i \preceq i$, para todo $i \in I$;

(2) Para todos $i, j, k \in I$, se $i \preceq j$ e $j \preceq k$, então $i \preceq k$;

(3) Para cada par $i, j$ de elementos em I, existe $k_{i, j}$ em I tal que $i \preceq k_{i, j}$ e $j \preceq k_{i, j}$.

Uma rede num conjunto $X$ é uma função $f: I \rightarrow X$, onde $I$ é um conjunto dirigido, denominado de conjunto de indices de $f$.

Observação 2.2. Para $i \in I$, dizemos que $f(i)$ é o i-ésimo termo de $f$. Analogamente à forma como denotamos as sequências, é usual representar o $i$-ésimo termo de $f$ por $x_{i}$. A rede $f$ também pode ser denotada por $\left(x_{i}\right)_{i \in I}$.

As redes, definidas dessa forma, nos permitem estender a noção de convergência de sequências, antes aplicável apenas aos espaços topológicos satisfazendo o primeiro axioma de enumerabilidade, agora para espaços topológicos em geral, por meio da noção de convergência de redes, que definimos abaixo.

Definição 2.3 ([Meg12, p. 144]). Seja $\left(x_{i}\right)_{i \in I}$ uma rede em um espaço topológico $X$ e seja $x$ um elemento de $X$. Dizemos que $\left(x_{i}\right)_{i \in I}$ converge para $x$ se, para todo aberto $U$ contendo $x$, existe $i_{U} \in I$ tal que $x_{i} \in U$ para qualquer $i \in I$ satisfazendo $i_{U} \preceq i$. Nesse caso $x$ é chamado de limite de $\left(x_{i}\right)_{i \in I}$ e usamos a notação $x_{i} \rightarrow x$ ou $\lim _{i \in I} x_{i}=x$.

Assim, muitos dos resultados aplicáveis a sequências possuem uma versão análoga para redes. São eles:

Proposição 2.4 ([Meg12, p. 145]). Um espaço topológico X é de Hausdorff se, e somente se, vale a unicidade do limite para cada rede convergente em $X$. 
Proposição 2.5 ([Meg12, p. 146]). Seja S um subconjunto de um espaço topológico X e seja $x$ um elemento de $X$. Então $x \in \bar{S}$ se, e somente se, existe uma rede em $S$ convergindo para $x$.

Proposição 2.6 ([Meg12, p. 146]). Um subconjunto $S$ de um espaço topológico $X$ é fechado se, $e$ somente se, $S$ contém os limites de todas as redes em $S$ convergentes.

Proposição 2.7 ([Meg12, p. 146]). Sejam X e $Y$ espaços topológicos e seja $f$ uma função de $X$ em $Y$.

(a) A função $f$ é contínua em $x_{0} \in X$ se, e somente se, $f\left(x_{i}\right) \rightarrow f\left(x_{0}\right)$ para toda rede $\left(x_{i}\right)_{i \in I}$ convergindo para $x_{0}$.

(b) A função $f$ é contínua em $X$ se, e somente se, para todo $x \in X$ e para toda rede $\left(x_{i}\right)_{i \in I}$ convergindo para $x$, temos $f\left(x_{i}\right) \rightarrow f(x)$.

A seguir, introduzimos o conceito de sub-rede, que generaliza o conceito de subsequência.

Definição 2.8 ([Meg12, p. 147]). Um subconjunto $J$ de um conjunto dirigido I é cofinal em I se para cada $\alpha \in I$, existe $\beta_{\alpha} \in J$ de modo que $\alpha \preceq \beta_{\alpha}$.

Definição 2.9 ([Meg12, p. 148]). Suponhamos que $X$ seja um conjunto, I seja um conjunto dirigido e suponhamos que $f: I \longrightarrow X$ seja uma rede. Além disso, suponhamos que $J$ seja um conjunto dirigido e $g: J \longrightarrow I$ seja uma função de maneira que

(1) Para todos $\beta_{1}, \beta_{2} \in J$, se $\beta_{1} \preceq \beta_{2}$, então $g\left(\beta_{1}\right) \preceq g\left(\beta_{2}\right)$ em I;

(2) $g(J)$ é cofinal em $I$.

Então a rede $f \circ g: J \longrightarrow X$ é chamada sub-rede de $f$.

Proposição 2.10 ([Meg12, p. 150]). Seja I um conjunto dirigido e $\left(x_{i}\right)_{i \in I}$ uma rede em $X$.

(a) A rede $\left(x_{i}\right)_{i \in I}$ é sub-rede de si mesma;

(b) Toda sub-rede de $\left(x_{i}\right)_{i \in I}$ é uma rede em $X$;

(c) Toda sub-rede de uma sub-rede de $\left(x_{i}\right)_{i \in I}$ é também uma sub-rede de $\left(x_{i}\right)_{i \in I}$;

(d) Se $X$ é um espaço topológico e $\left(x_{i}\right)_{i \in I}$ converge para um elemento $x \in X$, então toda sub-rede de $\left(x_{i}\right)_{i \in I}$ converge para $x$;

(e) Se $X$ é um espaço topológico e existe um elemento $x \in X$ tal que toda sub-rede de $\left(x_{i}\right)_{i \in I}$ tem uma sub-rede convergindo para $x$, então $x_{i} \rightarrow x$.

Proposição 2.11 ([Meg12, p. 152]). Um subconjunto $S$ de um espaço topológico $X$ é compacto se, e somente se, toda rede em $S$ admite uma sub-rede convergente com o limite em $S$.

Também faremos uso das noções de limsup e liminf de redes de números reais.

Definição 2.12 ([Meg12, p. 217]). Seja $\left(t_{i}\right)_{i \in I}$ uma rede em $\mathbb{R}$. Definimos o $\lim \inf$ de $\left(t_{i}\right)_{i \in I}$ por

$$
\liminf _{i \in I} t_{i}=\lim _{i \in I} \inf \left\{t_{j}: i \preceq j\right\},
$$

e o limsup de $\left(t_{i}\right)_{i \in I}$ por

$$
\limsup _{i \in I} t_{i}=\lim _{i \in I} \sup \left\{t_{j}: i \preceq j\right\} .
$$


Proposição 2.13 ([Meg12, p. 221-222]). Suponha que $\left(t_{i}\right)_{i \in I}$ e $\left(u_{i}\right)_{i \in I}$ são redes em $\mathbb{R}$ contendo o mesmo conjunto de índices I. Então:

(a) $\liminf _{i \in I} t_{i}=\sup \left\{\inf \left\{t_{j}: i \preceq j\right\}: i \in I\right\} \quad e \limsup _{i \in I} t_{i}=\inf \left\{\sup \left\{t_{j}: i \preceq j\right\}: i \in I\right\}$.

(b) $\liminf _{i \in I} t_{i} \leq \limsup _{i \in I} t_{i}$

(c) Se $s \geq 0$, então $\liminf _{i \in I} s t_{i}=s \liminf _{i \in I} t_{i} \quad e \limsup _{i \in I} s t_{i}=s \limsup _{i \in I} t_{i}$.

(d) Se $s \leq 0$, então $\liminf _{i \in I} s t_{i}=s \limsup _{i \in I} t_{i} \quad e \quad \limsup _{i \in I} s t_{i}=s \liminf _{i \in I} t_{i}$.

(e) $\liminf _{i \in I} t_{i}+\liminf _{i \in I} u_{i} \leq \liminf _{i \in I} t_{i}+u_{i} \quad e \quad \limsup _{i \in I} t_{i}+u_{i} \leq \limsup _{i \in I} t_{i}+\limsup _{i \in I} u_{i}$.

(f) $\liminf _{i \in I} t_{i}=\limsup _{i \in I} t_{i}$ se, e somente se, $\lim _{i \in I} t_{i}$ existe. Nesse caso, $\liminf _{i \in I} t_{i}=\limsup _{i \in I} t_{i}=\lim _{i \in I} t_{i}$.

Essas noções sobre redes são suficientes para entender este trabalho. É possível encontrar exemplos e mais resultados interessantes em [Meg12].

\subsection{Construindo funções}

Em muitos de nossos resultados será necessário tomar funções em $C_{0}(K, X)$ satisfazendo propriedades específicas. Nesta seção, enunciamos o Lema de Urysohn, resultado-chave que nos permite a construção de funções em $C_{0}(K, X)$, e posteriormente observamos algumas construções possíveis.

Teorema 2.14 (Lema de Urysohn [Rud87, p. 39]). Suponha que K é um espaço de Hausdorff localmente compacto, $U$ é um aberto de $K, P \subset U$ e $P$ é compacto. Então, existe uma função $f \in C_{0}(K)$, tal que $\operatorname{Im}(f) \subset[0,1],\left.f\right|_{U^{c}} \equiv 0$ e $\left.f\right|_{P} \equiv 1$.

No lema abaixo, sintetizamos todas as possíveis construções de funções em $C_{0}(K)$ e $C_{0}(K, X)$ a serem feitas ao longo deste trabalho.

Lema 2.15. Seja $K$ um espaço localmente compacto e Hausdorff e $k_{1}, \ldots, k_{n} \in K$ pontos dois a dois distintos e $\mathrm{v}_{1}, \ldots, \mathrm{v}_{n} \in X$. Então:

(1) Existem funções $\rho_{1}, \ldots, \rho_{n} \in C_{0}(K)$ tais que

(a) $\left\|\rho_{i}\right\|=1$, para todo $i \in I$;

(b) $\rho_{i}\left(k_{i}\right)=1$, para todo $i \in I$;

(c) Os suportes de $\rho_{i}$ são dois a dois disjuntos;

(2) Definindo $f_{i}(\cdot)=\rho_{i}(\cdot) \mathrm{v}_{i} \in C_{0}(K, X)$ para cada $i \in\{1, \ldots, n\}$, temos que

(a) $\left\|f_{i}\right\|=\mathrm{v}_{i}$, para todo $i \in I$;

(b) $f_{i}\left(k_{i}\right)=\mathrm{v}_{i}$, para todo $i \in I$;

(c) Os suportes de $f_{i}$ são dois a dois disjuntos;

(3) Para $f=f_{1}+\ldots+f_{n}$, temos $f\left(k_{i}\right)=\mathrm{v}_{i}$, para todo $i \in\{1, \ldots, n\}$ e $\|f\|=\max \left\{\left\|\mathrm{v}_{1}\right\|, \ldots,\left\|\mathrm{v}_{n}\right\|\right\}$.

Demonstração. Primeiramente, vejamos que é possível obter $U_{1}, \ldots, U_{n}$ vizinhanças de $k_{1}, \ldots, k_{n}$, respectivamente, de modo que $\overline{U_{1}}, \ldots, \overline{U_{n}}$ são dois a dois disjuntos.

De fato, por $K$ ser Hausdorff, podemos fixar $V_{1}, \ldots, V_{n}$ vizinhanças abertas de $k_{1}, \ldots, k_{n}$, respectivamente, de modo que sejam duas a duas disjuntas. Em seguida, como todo ponto de um espaço localmente compacto Hausdorff admite um sistema fundamental de vizinhanças compactas, podemos tomar, para cada $i \in\{1, \ldots, n\}$, um aberto $U_{i}$ de modo que $\overline{U_{i}}$ é compacto e $k_{i} \in U_{i} \subset \bar{U}_{i} \subset V_{i}$.

(1) Para cada $i \in\{1, \ldots, n\}$, aplicamos o Lema de Urysohn de modo a obter $\rho_{i} \in C_{0}(K)$ tal que $\operatorname{Im}\left(\rho_{i}\right) \subset[0,1],\left.f\right|_{U_{i}^{\mathrm{c}}} \equiv 0$ e $\left.\rho_{i}\right|_{\left\{k_{i}\right\}} \equiv 1$. Disso seguem as propriedades (a) e (b). Por fim, note que o suporte de $\rho_{i}$ está contido em $\overline{U_{i}}$, e sendo $\overline{U_{1}}, \ldots, \overline{U_{n}}$ dois a dois disjuntos, segue (c).

Os itens (2) e (3) são facilmente verificáveis a partir de (1). 


\subsection{Sobre a constante de Schäffer}

O parâmetro geométrico $\mathcal{S}(X)$, presente nas hipóteses do Teorema 1.16, é a constante de Schäffer de $X$ [Sch76], definida como

$$
\mathcal{S}(X)=\inf \{\max \{\|x+y\|,\|x-y\|\}:\|x\|=\|y\|=1\} .
$$

Por outro lado, há outro tipo de parâmetro geométrico de $X$, introduzido em [Jam64], definido por

$$
\mathcal{J}(X)=\sup \{\min \{\|x+y\|,\|x-y\|\}:\|x\|=\|y\|=1\},
$$

denominado constante de James de $X$.

Em [GL90], Gao \& Lau fizeram um extenso estudo sobre as constantes $\mathcal{S}(X)$ e $\mathcal{J}(X)$. Na proposição a seguir, listamos e damos referências às propriedades mais importantes.

Proposição 2.16. Seja $X$ um espaço de Banach de dimensão finita. Então:

(1) Se $X=\mathbb{R}$, então $\mathcal{S}(\mathbb{R})=2$ e $\mathcal{J}(\mathbb{R})=0$;

(2) Se $\operatorname{dim} X>1$, então $1 \leq \mathcal{S}(X) \leq \sqrt{2} \leq \mathcal{J}(X) \leq 2$ e $\mathcal{J}(X) \mathcal{S}(X)=2$;

(3) Se $X=\ell_{p}^{n}$, para certos $1<p<\infty$ e $n \geq 2$, então $\mathcal{S}\left(\ell_{p}^{n}\right)=\min \left\{2^{1 / p}, 2^{1-1 / p}\right\}$ e $\mathcal{J}\left(\ell_{p}^{n}\right)=$ $\max \left\{2^{1 / p}, 2^{1-1 / p}\right\}$;

(4) Se $X$ é um espaço de Hilbert, então $\mathcal{S}(X)=\mathcal{J}(X)=\sqrt{2}$;

Demonstração. O item (1) é imediato. Os itens (2) e (3) correspondem aos Teoremas 2.5 e 3.1 de [GL90]. Para verificar o item (4), note, pela regra do paralelogramo, que, para quaisquer $x, y \in X$ unitários, temos:

$$
\|x+y\|^{2}+\|x-y\|^{2}=2\|x\|^{2}+2\|x\|^{2}=4,
$$

donde segue que

$$
2(\min \{\|x+y\|,\|x-y\|\})^{2} \leq 4 \Rightarrow \min \{\|x+y\|,\|x-y\|\} \leq \sqrt{2}, \forall\|x\|=\|y\|=1,
$$

$\operatorname{logo}, \mathcal{J}(X) \leq \sqrt{2}$ que, junto às desigualdades de $(2)$, nos dá $\mathcal{J}(X)=\sqrt{2}$.

Analogamente, concluímos que

$$
\max \{\|x+y\|,\|x-y\|\} \geq \sqrt{2}, \forall\|x\|=\|y\|=1,
$$

$\operatorname{logo}, \mathcal{S}(X) \geq \sqrt{2}$ que, junto à desigualdade de $(2)$, nos dá $\mathcal{S}(X)=\sqrt{2}$.

A seguir, provamos uma estimativa com relação à constante de Schäffer de $X$ que será importante para a demonstração do Teorema 1.16.

Lema 2.17. Seja $X$ um espaço de Banach e $x, y \in X$. Então,

$$
\min \{\|x+y\|,\|x-y\|\} \leq \frac{2}{\mathcal{S}(X)} \max \{\|x\|,\|y\|\}
$$

Demonstração. Primeiro assuma que $X=\mathbb{R}$. Então $\mathcal{S}(X)=2$ e para cada par $x, y \in \mathbb{R}$, temos

$$
\min \{|x+y|,|x-y|\}=|| x|-| y|| \leq \max \{|x|,|y|\},
$$

e a prova está completa para este caso.

Suponha agora que $X$ tem dimensão pelo menos 2 e fixe $x, y \in X$. No caso em que $x=0$ ou $y=0$, usando que $\mathcal{S}(X) \leq 2$, o lema segue de imediato. Portanto, assumimos que ambos $x, y$ são não-nulos.

Faça $\hat{x}=x /\|x\|, \hat{y}=y /\|y\|$. A desigualdade que queremos provar não se altera ao permutarmos $x$ e $y$, e nem ao permutarmos $x$ e $-x$, logo, podemos assumir que

$$
\|x\|=\max \{\|x\|,\|y\|\} \text { e }\|\hat{x}+\hat{y}\|=\min \{\|\hat{x}+\hat{y}\|,\|\hat{x}-\hat{y}\|\} .
$$


Tendo em vista a definição de $\mathcal{J}(X)$ e o item (2) da Proposição 2.16, inferimos que

$$
\|\hat{x}+\hat{y}\|=\min \{\|\hat{x}+\hat{y}\|,\|\hat{x}-\hat{y}\|\} \leq \mathcal{J}(X)=\frac{2}{\mathcal{S}(X)} .
$$

Fazendo $\alpha=\|y\| /\|x\| \in(0,1]$ e usando que $\mathcal{S}(X) \leq 2$, temos

$$
\frac{\|x+y\|}{\|x\|}=\|\hat{x}+\alpha \hat{y}\| \leq(1-\alpha)\|\hat{x}\|+\alpha\|\hat{x}+\hat{y}\| \leq(1-\alpha) \frac{2}{\mathcal{S}(X)}+\alpha \frac{2}{\mathcal{S}(X)}=\frac{2}{\mathcal{S}(X)},
$$

e finalizamos a prova. 



\section{Capítulo 3}

\section{Os conjuntos de aproximação}

Neste capítulo introduzimos a técnica dos conjuntos de aproximação em sua forma mais geral. A partir de uma $(M, L)$-quasi-isometria bijetora $T: C_{0}(K, X) \rightarrow C_{0}(S, X) \operatorname{com} M<\sqrt{2}$, definimos os conjuntos de aproximação (Definições 3.1 e 3.2). Em seguida, apresentamos os Lemas da Existência (Lema 3.6) e da Simetria (Lema 3.7), que são peças-chave da técnica. Encerramos o capítulo enunciando a hipótese fundamental (Definição 3.8), sob a qual provamos a propriedade fundamental (Proposição 3.9), a qual garante a existência de um homeomorfismo $\varphi: K \rightarrow S$.

Tendo os resultados deste capítulo em mãos, nos próximos capítulos nos concentraremos apenas em provar que a hipótese fundamental é satisfeita para cada contexto particular.

\subsection{Definição dos conjuntos de aproximação}

A partir de agora, fixamos dois espaços de Hausdorff localmente compactos $K$ e $S$, um espaço de Banach de dimensão finita $X$ e uma $(M, L)$-quasi-isometria $T: C_{0}(K, X) \rightarrow C_{0}(S, X)$ bijetora, isto é, uma bijeção satisfazendo as desigualdades (Definição 1.4):

$$
\frac{1}{M}\|f-g\|-L \leq\|T(f)-T(g)\| \leq M\|f-g\|+L,
$$

para quaisquer $f, g \in C_{0}(K, X)$. Em virtude de $T$ ser bijetora, a hipótese de $\xi$-densidade da imagem, presente na Definição 1.4, é automaticamente satisfeita, razão pela qual a ocultamos adiante. Além disso, para que os resultados deste capítulo sejam válidos, devemos supor que $M<\sqrt{2}$.

Façamos alguns ajustes na quasi-isometria $T$. Primeiramente, podemos assumir que $T(0)=0$. De fato, note que a função $\hat{T}: C_{0}(K, X) \rightarrow C_{0}(S, X)$ dada por $\hat{T}(f)=T(f)-T(0)$ é uma $(M, L)$ quasi-isometria bijetora, com as mesmas constantes $M$ e $L$ de $T$ e satisfaz $\hat{T}(0)=0$. Além disso, podemos supor que ambas $T$ e $T^{-1}$ são $(M, L)$-quasi-isometrias, com as mesmas constantes $M$ e $L$. De fato, note que o fato de $T$ ser uma $(M, L)$-quasi-isometria implica que $T^{-1}$ é uma $(M, M L)$ quasi-isometria. Pondo $L^{\prime}=M L \geq L$, segue que ambas $T$ e $T^{-1}$ são $\left(M, L^{\prime}\right)$-quasi-isometrias.

Os conjuntos de aproximação $\Gamma_{\mathrm{w}}(k, \mathrm{v})$ são subconjuntos de $S$ formados por pontos que satisfazem uma propriedade de aproximação de funções através de $T$. Apresentamos abaixo sua definição formal e posteriormente uma digressão geométrica a fim de ilustrá-la.

No intuito de não sobrecarregar o texto, introduzimos a seguinte notação. Para cada $k \in K$, $f \in C_{0}(K, X)$ e v $\in X$, definimos:

$$
\omega(k, f, \mathrm{v})=\max \{\|f\|,\|f(k)-\mathrm{v}\|\} .
$$

Como ilustração, considerando $X=\mathbb{R}$ e $K=(0,1)$, para cada $k \in K, f \in C_{0}(K)$ e v $\in \mathbb{R}$, $\omega(k, f, \mathrm{v})$ representa o máximo das distâncias destacadas abaixo: 


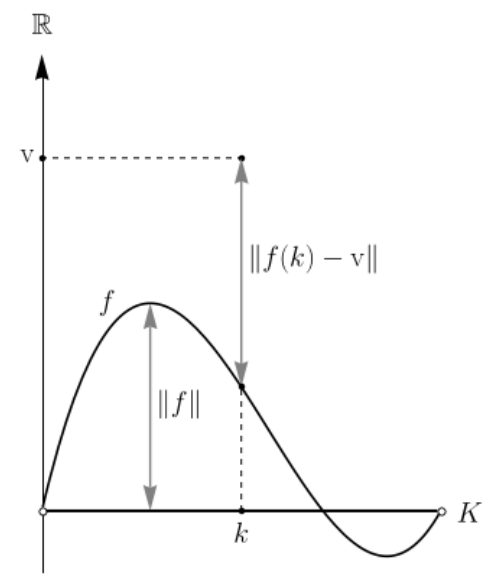

Definição 3.1. Sejam $k \in K, \mathrm{v}, \mathrm{w} \in X$ tais que $\|\mathrm{v}\| \geq M L$ e $\|\mathrm{w}\|=\|\mathrm{v}\| / M-L$. Definimos

$$
\Gamma_{\mathrm{w}}(k, \mathrm{v})=\left\{s \in S:\|T f(s)-\mathrm{w}\| \leq M \omega(k, f, \mathrm{v})+L, \forall f \in C_{0}(K, X)\right\} .
$$

Destacamos alguns detalhes da definição acima. Inicialmente, não há resultados que nos garantam que os conjuntos $\Gamma_{\mathrm{w}}(k, \mathrm{v})$ definidos acima sejam não-vazios. Essa lacuna será preenchida na seção 3.2, com o Lema 3.6, o qual nos garantirá que $\Gamma_{\mathrm{w}}(k, \mathrm{v}) \neq \emptyset$ quando $\|\mathrm{v}\|$ for suficientemente grande, razão pela qual o chamaremos de lema da existência. A hipótese ' $\|\mathrm{w}\|=\|\mathrm{v}\| / M-L$ ', que a priori parece arbitrária, estará justificada pela demonstração desse lema, bem como o termo ' $\omega(k, f, \mathrm{v})$ '.

Vejamos abaixo uma ilustração geométrica da propriedade satisfeita pelos pontos $s \in \Gamma_{\mathrm{w}}(k, \mathrm{v})$, considerando $K=S=(0,1)$ e $X=\mathbb{R}$.
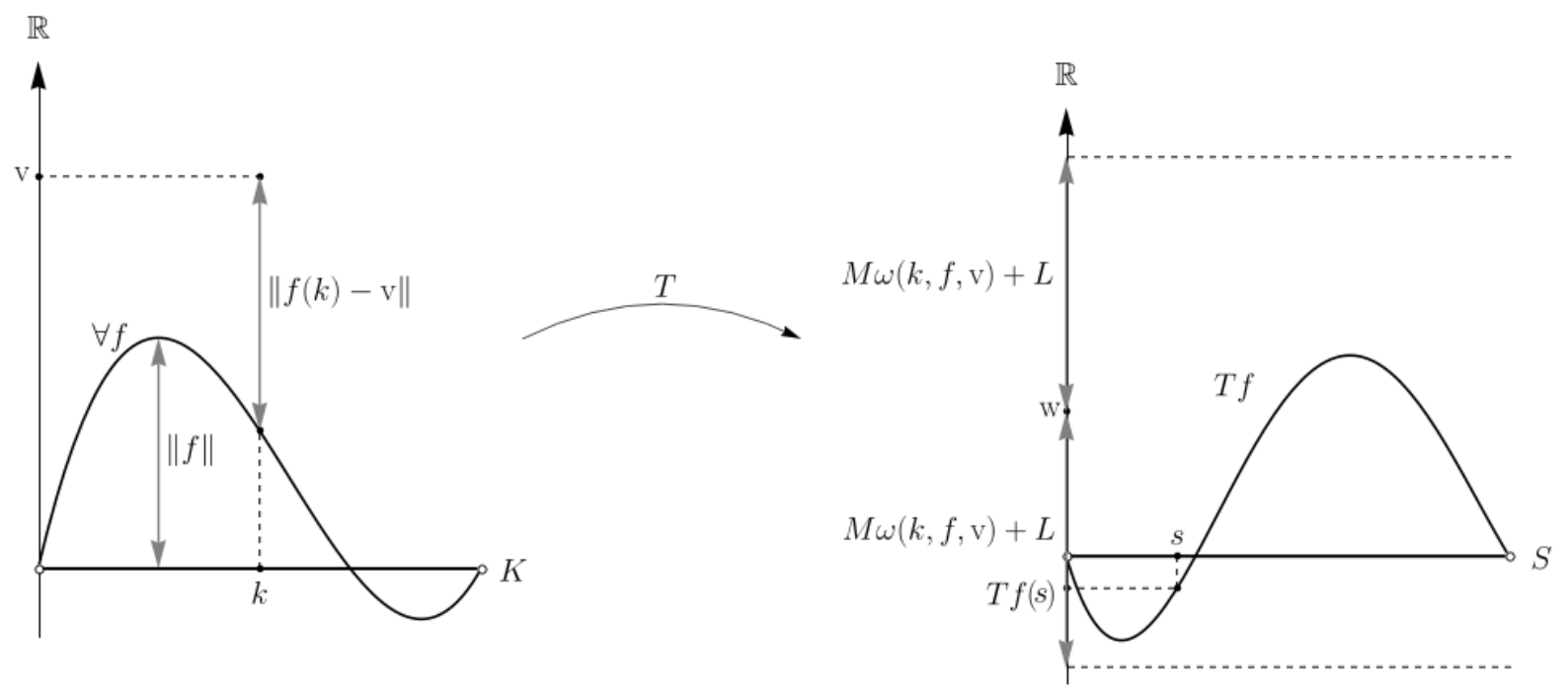

Observe que os conjuntos $\Gamma_{\mathrm{w}}(k, \mathrm{v})$ estão em função da quasi-isometria $T$ fixada no início do capítulo. Sendo $T$ bijetora, podemos considerar a função $T^{-1}$, que também é uma $(M, L)$-quasiisometria. Assim, é possível definirmos os conjuntos de aproximação com respeito a $T^{-1}$ em vez de $T$, como expomos a seguir.

Primeiramente, estendemos a notação introduzida em (3.1) para $C_{0}(S, X)$, isto é, para cada $s \in S, g \in C_{0}(S, X)$ e w $\in X$, definimos:

$$
\omega(s, g, \mathrm{w})=\max \{\|g\|,\|g(s)-\mathrm{w}\|\} .
$$

A seguir, definimos os conjuntos de aproximação com relação a $T^{-1}$, os quais denotamos por ' $\Lambda$ ' ao 
invés de ' $\Gamma$ ', para evitar confusões de notação.

Definição 3.2. Sejam $s \in S, \mathrm{w}, \mathrm{z} \in X$ tais que $\|\mathrm{w}\| \geq M L e\|\mathrm{z}\|=\|\mathrm{w}\| / M-L$. Definimos

$$
\Lambda_{\mathrm{z}}(s, \mathrm{w})=\left\{k \in K:\left\|T^{-1} g(k)-\mathrm{z}\right\| \leq M \omega(s, g, \mathrm{w})+L, \forall g \in C_{0}(S, X)\right\} .
$$

A interpretação geométrica dos conjuntos $\Lambda_{\mathrm{z}}(s, \mathrm{w})$ é análoga à dos conjuntos $\Gamma_{\mathrm{w}}(k, \mathrm{v})$. Mais ainda, sendo $T$ e $T^{-1}$ ambas $(M, L)$-quasi-isometrias, com as mesmas constantes $M$ e $L$, todos os resultados provados neste capítulo para os conjuntos $\Gamma_{\mathrm{w}}(k, \mathrm{v})$ têm uma versão análoga para os conjuntos $\Lambda_{\mathrm{z}}(s, \mathrm{w})$, à qual nos referiremos como sendo a sua respectiva versão simétrica.

Já que nas definições dos conjuntos $\Gamma_{\mathrm{w}}(k, \mathrm{v})$ e $\Lambda_{\mathrm{z}}(s, \mathrm{w})$ já é assumido que $\|\mathrm{v}\| \geq M$ e $\|\mathrm{w}\|=$ $\|\mathrm{v}\| / M-L$, respectivamente, $\|\mathrm{w}\| \geq M$ e $\|\mathrm{z}\|=\|\mathrm{w}\| / M-L$, tais restrições nas normas dos parâmetros estarão implícitas em cada uso desses conjuntos.

A ideia principal da técnica dos conjuntos de aproximação $\Gamma_{\mathrm{w}}(k, \mathrm{v})$ é construir uma função contínua $\varphi: K \rightarrow S$ a partir deles. Mais precisamente, dado $k \in K$, gostaríamos de definir $\varphi(k)$ como sendo um dos pontos de $\Gamma_{\mathrm{w}}(k, \mathrm{v})$, para certos v e w. Assim, para cada $k \in K$, os conjuntos $\Gamma_{\mathrm{w}}(k, \mathrm{v}) \subset S$ devem ser vistos como conjuntos de "candidatos a imagem de $k$ por $\varphi$ ".

A priori, os conjuntos $\Gamma_{\mathrm{w}}(k, \mathrm{v})$ podem conter mais do que um elemento. Portanto, a dificuldade na defininição $\varphi$ estaria em escolher continuamente, para cada $k \in K$, sua respectiva imagem em conjuntos $\Gamma_{\mathrm{w}}(k, \mathrm{v})$. A propriedade fundamental (a ser provada na seção 3.4), nos garantirá que, sob certas condições, existe uma função $\varphi: K \rightarrow S$ contínua dessa forma. Por simetria, podemos fazer uma construção análoga à de $\varphi$, agora com relação aos conjuntos $\Lambda_{\mathrm{z}}(s, \mathrm{w})$. Feito isso, teremos duas funções contínuas $\varphi: K \rightarrow S$ e $\psi: S \rightarrow K$ e a prova da propriedade fundamental é concluída mostrando que $\psi=\varphi^{-1}$.

\subsection{Lema da existência}

Vejamos abaixo a definição de redes $\mathrm{v}$-pico.

Definição 3.3. Dado $k \in K$, dizemos que uma rede $\left(\rho_{i}\right)_{i \in I} \in C_{0}(K)$ é uma rede pico em $k$ se:

(a) $\operatorname{Im} \rho_{i} \subset[0,1], \forall i \in I$;

(b) $\rho_{i}(k)=1, \forall i \in I$;

(c) Para toda $U$ vizinhança aberta de $k$, temos

$$
\lim _{i \in I}\left\|\rho_{i}\right\|_{K \backslash U}=0 .
$$

Então, para cada $\mathrm{v} \in X$ e $k \in K$, dizemos que $\left(f_{i}\right)_{i \in I} \in C_{0}(K, X)$ é uma rede $\mathrm{v}$-pico de $k$ se existe uma rede pico $\left(\rho_{i}\right)_{i \in I}$ em $k$ tal que $f_{i}(\cdot)=\rho_{i}(\cdot) \mathrm{v}$, para todo $i \in I$.

No caso $K=(0,1)$ e $X=\mathbb{R}$, as redes v-pico em $k$ possuem a propriedade de convergência ilustrada abaixo. 


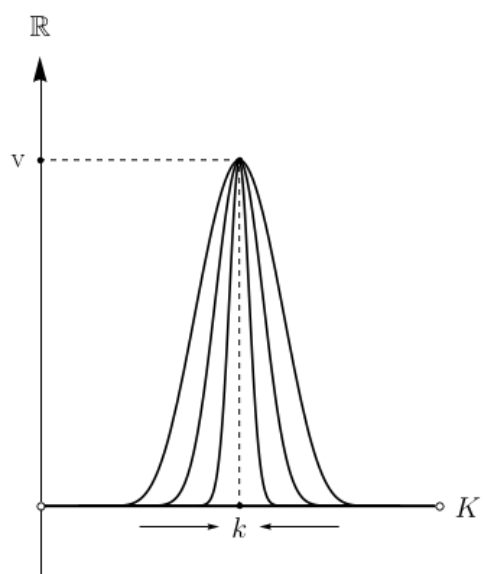

Considerando $\left(f_{i}\right)_{i \in I} \in C_{0}(K, X)$ uma rede v-pico de $k$ e $f \in C_{0}(K, X)$ escolhida arbitrariamente, a ilustração abaixo exemplifica o comportamento das funções $f-f_{i}$.
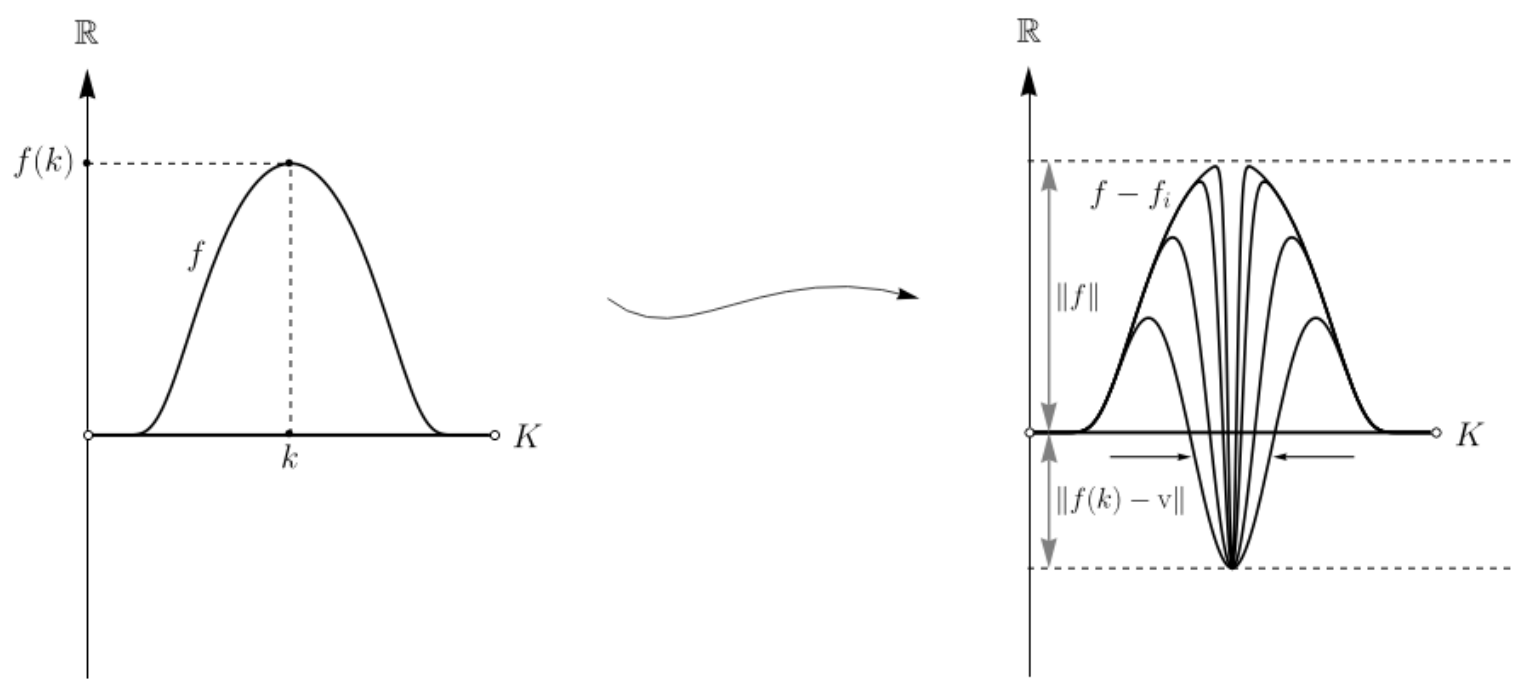

Observe que, na figura acima, a norma das funções $f-f_{i}$ estão limitadas pelo máximo entre $\|f\|$ e $\|f(k)-\mathrm{v}\|$, isto é, por $\omega(k, f, \mathrm{v})$. No exemplo acima, essa propriedade ocorre para todas as funções $f-f_{i}$, no entanto, em geral, apenas garantimos tal limitação para o lim sup das normas de $f-f_{i}$, precisamente o que provamos no lema a seguir.

Lema 3.4. Sejam $k \in K, \mathrm{v} \in X e\left(f_{i}\right)_{i \in I} \in C_{0}(K, X)$ uma rede v-pico para $k$. Então, para toda $f \in C_{0}(K, X)$, temos

$$
\limsup _{i \in I}\left\|f-f_{i}\right\| \leq \max \{\|f\|,\|f(k)-\mathrm{v}\|\}=\omega(k, f, \mathrm{v}) .
$$

Demonstração. Fixe $\varepsilon>0$ e, pela continuidade de $f$, tome $U_{\varepsilon}$ vizinhança aberta de $k$ tal que

$$
\|f(u)-f(k)\|<\varepsilon, \forall u \in U_{\varepsilon} .
$$

De acordo com a propriedade (c) das redes-pico (Definição 3.3), existe $i_{0} \in I$ tal que

$$
\left\|f_{i}\right\|_{K \backslash U_{\varepsilon}}=\|\mathrm{v}\|\left\|\rho_{i}\right\|_{K \backslash U_{\varepsilon}}<\varepsilon, \forall i \geq i_{0} .
$$

Para cada $i \geq i_{0}$, avaliamos $\left\|f(u)-f_{i}(u)\right\|$ em dois casos abaixo:

Caso 1. $u \in U_{\varepsilon}$.

Em virtude da propriedade (a) das redes-pico, segue que $\operatorname{Im}\left(f_{i}\right)$ está contida no segmento $\{t v: t \in$ 
$[0,1]\}$ que liga 0 a v. Portanto, tomando $\alpha \in[0,1]$ tal que $f_{i}(u)=\alpha \mathrm{v}$, obtemos

$$
\left\|f(u)-f_{i}(u)\right\| \leq(1-\alpha)\|f(u)\|+\alpha\|f(u)-\mathrm{v}\| \leq \max \{\|f\|,\|f(u)-\mathrm{v}\|\} .
$$

e então, por (3.2), segue que

$$
\left\|f(u)-f_{i}(u)\right\| \leq \max \{\|f\|,\|f(k)-\mathrm{v}\|\}+\varepsilon .
$$

Caso 2. $u \in K \backslash U_{\varepsilon}$.

Tendo em vista (3.3), note que

$$
\left\|f(u)-f_{i}(u)\right\| \leq\|f(u)\|+\left\|f_{i}(u)\right\| \leq\|f\|+\left\|f_{i}\right\|_{K \backslash U_{\varepsilon}}<\|f\|+\varepsilon .
$$

Segue dos casos 1 e 2 acima que

$$
\left\|f-f_{i}\right\| \leq \max \{\|f\|,\|f(k)-\mathrm{v}\|\}+\varepsilon, \forall i \geq i_{0} .
$$

Como para todo $\varepsilon>0$ existe $i_{0} \in I$ satisfazendo esta propriedade, segue que

$$
\limsup _{i \in I}\left\|f-f_{i}\right\| \leq \max \{\|f\|,\|f(k)-\mathrm{v}\|\} .
$$

Com o Lema 3.4 provado, estamos aptos à demonstração do lema da existência. Antes, vejamos uma característica dos números $\omega(k, f, \mathrm{v})$ da qual faremos uso frequentemente, tanto no lema da existência como no restante dos lemas.

Observação 3.5. Por definição, os números $\omega(k, f, \mathrm{v})$ são sempre maiores ou iguais que $\|f\|$. Em virtude disso, nem sempre estes números nos dão valores pequenos para a estimativa $M \omega(k, f, \mathrm{v})+L$, que consta na definição dos conjuntos $\Gamma_{\mathrm{w}}(k, \mathrm{v})$.

Nos resultados a frente, fazemos uso da situação minimal em que $\omega(k, f, \mathrm{v})=\|\mathrm{v}\| / 2$. Vejamos que tal situação corresponde precisamente ao caso em que $f$ satisfaz $\|f\|=\|\mathrm{v}\| / 2$ e $f(k)=\mathrm{v} / 2$.

De fato, para tal $f$, é claro que $\omega(k, f, \mathrm{v})=\|\mathrm{v}\| / 2$. O valor $\|\mathrm{v}\| / 2$ é o minimo valor possivel pois para $\omega(k, f, \mathrm{v})$, pois

$$
\|\mathrm{v}\| \leq\|f(k)\|+\|f(k)-\mathrm{v}\| \leq \omega(k, f, \mathrm{v})+\omega(k, f, \mathrm{v}) \quad \Rightarrow \quad \omega(k, f, \mathrm{v}) \geq \frac{\|\mathrm{v}\|}{2} .
$$

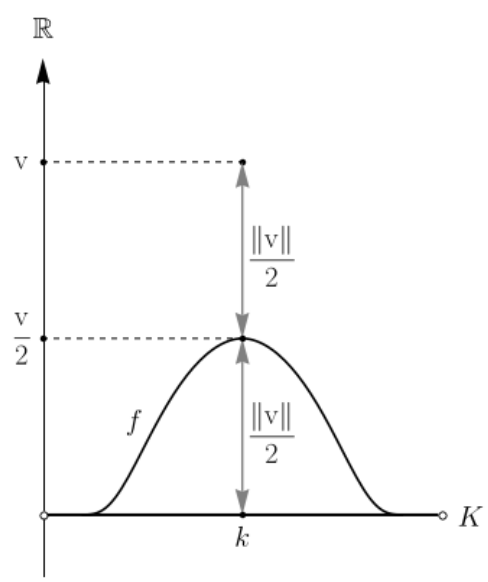

Lema 3.6 (Lema da existência). Existe $N_{0} \geq 0$, dependendo apenas de $M$ e L de modo que para todo $k \in K$ e $\mathrm{v} \in X$ com $\|\mathrm{v}\|>N_{0}$, existe $\mathrm{w} \in X$ tal que $\Gamma_{\mathrm{w}}(k, \mathrm{v}) \neq \emptyset$. Além disso, podemos tomar $N_{0}=0$ quando $L=0$. 
Demonstração. Fazendo uso do Lema de Urysohn (Teorema 2.14), fixemos $\left(f_{i}\right)_{i \in I}$ rede v-pico de $k$. Pela propriedade (b) das redes-pico, temos que $\left\|f_{i}\right\|=\|\mathrm{v}\|$ para todo $i \in I$, e portanto,

$$
\frac{\|\mathrm{v}\|}{M}-L \leq\left\|T f_{i}\right\| \leq M\|\mathrm{v}\|+L, \forall i \in I .
$$

Para cada $i \in I$, tomemos $s_{i} \in S$ tal que $\left\|T f_{i}\left(s_{i}\right)\right\|=\left\|T f_{i}\right\|$. Segue que

$$
\frac{\|\mathrm{v}\|}{M}-L \leq\left\|T f_{i}\left(s_{i}\right)\right\| \leq M\|\mathrm{v}\|+L, \forall i \in I .
$$

Em particular, $\left(T f_{i}\left(s_{i}\right)\right)_{i \in I}$ é uma rede limitada de $X$. Como $X$ tem dimensão finita, a rede limitada $\left(T f_{i}\left(s_{i}\right)\right)_{i \in I}$ admite sub-rede convergente e podemos supor que $T f_{i}\left(s_{i}\right) \rightarrow \mathrm{w}_{0}$. Por (3.4), segue que

$$
\left\|\mathrm{w}_{0}\right\| \geq \frac{\|\mathrm{v}\|}{M}-L
$$

Mais adiante, definiremos $\mathrm{w}$ a partir de $\mathrm{w}_{0}$.

Vejamos agora que $\left(s_{i}\right)_{i \in I}$ admite sub-rede convergente. De fato, para toda $f \in C_{0}(K, X)$, temos

$$
\left\|T f\left(s_{i}\right)-T f_{i}\left(s_{i}\right)\right\| \leq\left\|T f-T f_{i}\right\| \leq M\left\|f-f_{i}\right\|+L, \forall i \in I,
$$

logo, usando que $T f_{i}\left(s_{i}\right) \rightarrow \mathrm{w}_{0}$ e o Lema 3.4, concluímos que

$$
\begin{aligned}
\limsup _{i \in I}\left\|T f\left(s_{i}\right)-\mathrm{w}_{0}\right\| & =\limsup _{i \in I}\left\|T f\left(s_{i}\right)-T f_{i}\left(s_{i}\right)\right\| \\
& \leq \limsup _{i \in I} M\left\|f-f_{i}\right\|+L \\
& \leq M \omega(k, f, \mathrm{v})+L .
\end{aligned}
$$

Fixemos $f_{0} \in C_{0}(K, X)$ tal que $\left\|f_{0}\right\|=\|\mathrm{v}\| / 2$ e $f_{0}(k)=\mathrm{v} / 2$ (veja Lema 2.15.(2)). Esta é precisamente a situação minimal que discutimos na Observação 3.5. Temos que $\omega\left(k, f_{0}, \mathrm{v}\right)=\|\mathrm{v}\| / 2 \mathrm{e}$ tendo em vista (3.5) e (3.6), obtemos

$$
\begin{aligned}
\liminf _{i \in I}\left\|T f_{0}\left(s_{i}\right)\right\| & \geq \liminf _{i \in I}\left(\left\|\mathrm{w}_{0}\right\|-\left\|T f_{0}\left(s_{i}\right)-\mathrm{w}_{0}\right\|\right) \\
& =\left\|\mathrm{w}_{0}\right\|-\limsup _{i \in I}\left\|T f_{0}\left(s_{i}\right)-\mathrm{w}_{0}\right\| \\
& \geq\left(\frac{\|\mathrm{v}\|}{M}-L\right)-\left(M \frac{\|\mathrm{v}\|}{2}+L\right) \\
& =\|\mathrm{v}\|\left(\frac{1}{M}-\frac{M}{2}\right)-2 L .
\end{aligned}
$$

Por hipótese,

$$
M<\sqrt{2} \Rightarrow M^{2}<2 \Rightarrow \frac{M}{2}<\frac{1}{M} \Rightarrow \frac{1}{M}-\frac{M}{2}>0,
$$

logo, segue de (3.7) que para todo v satisfazendo

$$
\|\mathrm{v}\|>\frac{4 M L}{2-M^{2}}=N_{0},
$$

temos

$$
\liminf _{i \in I}\left\|T f_{0}\left(s_{i}\right)\right\|>0 .
$$

Como $T f_{0}$ se anula no infinito, a desigualdade acima implica que para $i_{0}$ suficientemente grande, $\left(s_{i}\right)_{i \geq i_{0}}$ está contida num compacto da forma $\left\{s \in S:\left\|T f_{0}(s)\right\| \geq \varepsilon\right\}$ para algum $\varepsilon>0$. Por consequência disso, $\left(s_{i}\right)_{i \in I}$ admite sub-rede convergente.

Podemos, portanto, supor que $s_{i} \rightarrow s$. Sendo assim, para toda $f \in C_{0}(K, X)$, temos que 
$T f\left(s_{i}\right) \rightarrow T f(s)$ e então segue de (3.6) que

$$
\left\|T f(s)-\mathrm{w}_{0}\right\| \leq M \omega(k, f, \mathrm{v})+L, \forall f \in C_{0}(K, X) .
$$

Nos resta agora "ajustar a norma" de $\mathrm{w}_{0}$ para que fique igual a $\|\mathrm{v}\| / M-L$. Defina $\mathrm{w}=\alpha \mathrm{w}_{0}$, onde $\alpha=\frac{\|\mathrm{v}\| / M-L}{\left\|\mathrm{w}_{0}\right\|}$. É claro que $\|\mathrm{w}\|=\|\mathrm{v}\| / M-L$.

Por fim, vejamos que $s \in \Gamma_{\mathrm{w}}(k, \mathrm{v})$. Fixe $f \in C_{0}(K, X)$. Note que, além de (3.9), temos

$$
\|T f(s)\| \leq\|T f\| \leq M\|f\|+L \leq M \omega(k, f, \mathrm{v})+L .
$$

Logo, tendo em vista que $0<\alpha \leq 1$, temos

$$
\|T f(s)-\mathrm{w}\| \leq(1-\alpha)\|T f(s)\|+\alpha\left\|T f(s)-\mathrm{w}_{0}\right\| \leq M \omega(k, f, \mathrm{v})+L .
$$

Como $f \in C_{0}(K, X)$ acima foi fixada arbitrariamente, segue que $s \in \Gamma_{\mathrm{w}}(k, \mathrm{v})$.

Para concluir que podemos tomar $N_{0}=0$ quando $L=0$, basta rever (3.8).

\subsection{Lema da simetria}

Um dos pontos-chave da técnica dos conjuntos de aproximação está em fazer uso da simetria existente entre os conjuntos $\Gamma_{\mathrm{w}}(k, \mathrm{v})$ e $\Lambda_{\mathrm{z}}(s, \mathrm{w})$, definidos com respeito a $T$ e $T^{-1}$, respectivamente. Como já observamos anteriormente, todos os resultados provados para os conjuntos $\Gamma_{\mathrm{w}}(k, \mathrm{v})$ também são válidos para $\Lambda_{\mathrm{z}}(s, \mathrm{w})$. Nesta seção vemos que, além disso, o fato de $T$ e $T^{-1}$ serem inversas uma da outra nos permite concluir um tipo de relação de invertibilidade entre os conjuntos $\Gamma_{\mathrm{w}}(k, \mathrm{v})$ e $\Lambda_{\mathrm{z}}(s, \mathrm{w})$, exposta na forma do lema a seguir.

Lema 3.7 (Lema da simetria). Existe $N_{1} \geq 0$, dependendo apenas de $M$ e $L$, de modo que para todo $k \in K$ e $\mathrm{v} \in X$ com $\|\mathrm{v}\|>N_{1}$, se $s \in \Gamma_{\mathrm{w}}(k, \mathrm{v})$ e $\Lambda_{\mathrm{z}}(s, \mathrm{w}) \neq \emptyset$, então $\Lambda_{\mathrm{z}}(s, \mathrm{w})=\{k\}$. Podemos tomar $N_{1}=0$ quando $L=0$.

Demonstração. Como $\Lambda_{\mathrm{z}}(s, \mathrm{w}) \neq \emptyset$, tome $k^{\prime} \in \Lambda_{\mathrm{z}}(s, \mathrm{w})$. Temos que provar que $k^{\prime}=k$. Suponha o contrário, então, pelo Lema 2.15.(3), existe $f_{0} \in C_{0}(K, X)$ satisfazendo:

$$
\left\|f_{0}\right\|=\|\mathrm{v}\| / 2, f_{0}(k)=\mathrm{v} / 2 \text { e } f_{0}\left(k^{\prime}\right)=-\frac{\|\mathrm{v}\|}{2} \frac{\mathrm{z}}{\|\mathrm{z}\|}
$$

Note que $\omega\left(k, f_{0}, \mathrm{v}\right)=\|\mathrm{v}\| / 2$, logo, aplicando a definição de $s \in \Gamma_{\mathrm{w}}(k, \mathrm{v})$ a $f_{0}$, temos

$$
\left\|T f_{0}(s)-\mathrm{w}\right\| \leq M \frac{\|\mathrm{v}\|}{2}+L .
$$

Além disso, como $\left\|f_{0}\right\| \leq\|\mathrm{v}\| / 2$, temos

$$
\left\|T f_{0}\right\| \leq M \frac{\|\mathrm{v}\|}{2}+L
$$

Portanto,

$$
\omega\left(s, T f_{0}, \mathrm{w}\right)=\max \left\{\left\|T f_{0}\right\|,\left\|T f_{0}(s)-\mathrm{w}\right\|\right\} \leq M \frac{\|\mathrm{v}\|}{2}+L,
$$

e, aplicando a definição de $k^{\prime} \in \Lambda_{\mathrm{z}}(s, \mathrm{w})$ para $T f_{0}$, obtemos

$$
\left\|f_{0}\left(k^{\prime}\right)-\mathrm{z}\right\| \leq\left\|T^{-1}\left(T f_{0}\right)\left(k^{\prime}\right)-\mathrm{z}\right\| \leq M \omega\left(s, T f_{0}, \mathrm{w}\right)+L \leq M\left(M \frac{\|\mathrm{v}\|}{2}+L\right)+L .
$$


Como $f_{0}\left(k^{\prime}\right)$ tem norma $\|\mathrm{v}\| / 2$ e sentido oposto ao de $\mathrm{z}$,

$$
\left\|f_{0}\left(k^{\prime}\right)-\mathrm{z}\right\|=\|\mathrm{v}\| / 2+\|\mathrm{z}\|=\|\mathrm{v}\| / 2+\|\mathrm{w}\| / M-L=\|\mathrm{v}\| / 2+(\|\mathrm{v}\| / M-L) / M-L,
$$

e, juntando as desigualdades (3.11) e (3.12) acima, obtemos

$$
\|\mathrm{v}\|\left(\frac{1}{2}+\frac{1}{M^{2}}-\frac{M^{2}}{2}\right) \leq \frac{L}{M}+2 L+M L
$$

mas note que

$$
\frac{1}{2}+\frac{1}{M^{2}}-\frac{M^{2}}{2}=\frac{M^{2}+2-M^{4}}{2 M^{2}}=\frac{\left(M^{2}+1\right)\left(2-M^{2}\right)}{2 M^{2}}>0,
$$

ja que, por hipótese, $M<\sqrt{2}$. Portanto, quando

$$
\|\mathrm{v}\|>\frac{\frac{L}{M}+2 L+M L}{\frac{1}{2}+\frac{1}{M^{2}}-\frac{M^{2}}{2}}=\frac{1+2 M+M^{2}}{\left(M^{2}+1\right)\left(2-M^{2}\right)} 2 M L=N_{1},
$$

obtemos uma contradição. Além disso, da desigualdade acima segue que podemos tomar $N_{1}=0$ quando $L=0$.

\subsection{A propriedade fundamental dos conjuntos de aproximação}

Nesta seção provamos a Proposição 3.9 que nos permite reduzir a demonstração dos Teoremas 1.11 e 1.24 à verificação da hipótese fundamental, definida abaixo.

Definição 3.8 (Hipótese fundamental). Para cada $k \in K, s \in S$ e $N \geq M L$, definimos:

$$
\Phi_{N}(k)=\bigcup\left\{\Gamma_{\mathrm{w}}(k, \mathrm{v}):\|\mathrm{v}\|>N e\|\mathrm{w}\|=\|\mathrm{v}\| / M-L\right\},
$$

$e$

$$
\Psi_{N}(s)=\bigcup\left\{\Lambda_{\mathrm{z}}(s, \mathrm{w}):\|\mathrm{w}\|>N e\|\mathrm{z}\|=\|\mathrm{w}\| / M-L\right\} .
$$

Dizemos que $N \geq M L$ satisfaz a hipótese fundamental se para todo $k \in K$ e todo $s \in S$ os conjuntos $\Phi_{N}(k)$ e $\Psi_{N}(s)$ são unitários.

Proposição 3.9 (Propriedade fundamental). Se $N \geq M L$ satisfaz a hipótese fundamental, então a função $\varphi: K \rightarrow S$ dada por $\Phi_{N}(k)=\{\varphi(k)\}$ é um homeomorfismo.

Antes de provarmos a Proposição acima, estabelecemos abaixo algumas convenções e resultados importantes. Nos resultados expostos acima, fixamos constantes $N_{0}$ e $N_{1}$, dadas respectivamente pelos Lemas 3.6 e 3.7, e uma constante $N$ satisfazendo a hipótese fundamental. Definimos a constante auxiliar

$$
N^{\prime}=\max \left\{N_{0}, N_{1}, N\right\} .
$$

Conforme já foi feito no enunciado da Proposição 3.9, podemos definir uma função $\varphi: K \rightarrow S$ dada por $\Phi_{N}(k)=\{\varphi(k)\}$. Além disso, podemos definir a versão simétrica de $\varphi$, isto é, $\psi: S \rightarrow K$ dada por $\Psi_{N}(s)=\{\psi(s)\}$.

O Lema abaixo será utilizado na demonstração da Proposição 3.9.

Lema 3.10. Para todo $k \in K$ e $\mathrm{v} \in X$ com $\|\mathrm{v}\|>N^{\prime}$, existe $\mathrm{w} \in X$ tal que $\Gamma_{\mathrm{w}}(k, \mathrm{v})=\{\varphi(k)\}$.

Demonstração. Fixe $k \in K$ e v $\in X$ com $\|\mathrm{v}\|>N^{\prime}$. Como $\|\mathrm{v}\|>N^{\prime} \geq N_{0}$, pelo lema da existência, fixemos w tal que

$$
\Gamma_{\mathrm{w}}(k, \mathrm{v}) \neq \emptyset .
$$


Por outro lado, como $\|\mathrm{v}\|>N$, segue da definição de $\Phi_{N}(k)$ que

$$
\Gamma_{\mathrm{w}}(k, \mathrm{v}) \subset \Phi_{N}(k)=\{\varphi(k)\} .
$$

Por (3.13) e (3.14) concluímos que $\Gamma_{\mathrm{w}}(k, \mathrm{v})=\{\varphi(k)\}$.

Com isso, estamos aptos a provar a propriedade fundamental abaixo.

Demonstração da Proposição 3.9. A demonstração é feita mediante os dois passos a seguir.

Passo 1. A função $\varphi: K \rightarrow S$ é contínua.

Tome uma rede $\left(k_{i}\right)_{i \in I} \subset K$ tal que $k_{i} \rightarrow k$. Temos que provar que $\varphi\left(k_{i}\right) \rightarrow \varphi(k)$. Para tal, é suficiente verificarmos que

$$
\left(\varphi\left(k_{i}\right)\right)_{i \in I} \text { admite uma sub-rede convergente à } \varphi(k) .
$$

De fato, uma vez provado (3.15), como $k_{i} \rightarrow k$ foi fixada de modo arbitrário, esse fato também é válido para toda sub-rede de $\left(k_{i}\right)_{i \in I} \subset K$. Segue disso que toda sub-rede de $\left(\varphi\left(k_{i}\right)\right)_{i \in I}$ admite uma sub-rede convergente à $\varphi(k)$, e consequentemente, $\varphi\left(k_{i}\right) \rightarrow \varphi(k)$ (veja o Lema 2.10.(e)).

Para verificarmos (3.15), primeiramente, vemos que $\varphi\left(k_{i}\right)$ admite sub-rede convergente a um certo $s \in S$ e, em seguida, que $s=\varphi(k)$.

Fixe $\mathrm{v} \in X$ com $\|\mathrm{v}\|>N^{\prime}$. Pelo Lema 3.10, tomemos para cada $i \in I, \mathrm{w}_{i} \in X$ tal que $\left\|\mathrm{w}_{i}\right\|=\|\mathrm{v}\| / M-L$ e $\Gamma_{\mathrm{w}_{i}}\left(k_{i}, \mathrm{v}\right)=\left\{\varphi\left(k_{i}\right)\right\}$. Como os $\mathrm{w}_{i}$ 's têm todos a mesma norma, segue em particular que $\left(\mathrm{w}_{i}\right)_{i \in I}$ é limitada e pelo fato de $X$ ter dimensão finita, $\left(\mathrm{w}_{i}\right)_{i \in I}$ admite sub-rede convergente a um certo $\mathrm{w} \in X$ satisfazendo $\|\mathrm{w}\|=\|\mathrm{v}\| / M-L$. Podemos, então, supor que $\mathrm{w}_{i} \rightarrow \mathrm{w}$. Dada $f \in C_{0}(K, X)$, como $\varphi\left(k_{i}\right) \in \Gamma_{\mathrm{w}_{i}}\left(k_{i}, \mathrm{v}\right)$, temos que

$$
\left\|T f\left(\varphi\left(k_{i}\right)\right)-\mathrm{w}_{i}\right\| \leq M \omega\left(k_{i}, f, \mathrm{v}\right)+L, \forall i \in I
$$

e tomando o limsup acima, concluímos que:

$$
\begin{aligned}
\limsup _{i \in I}\left\|T f\left(\varphi\left(k_{i}\right)\right)-\mathrm{w}\right\| & =\underset{i \in I}{\limsup }\left\|T f\left(\varphi\left(k_{i}\right)\right)-\mathrm{w}_{i}\right\| \\
& \leq \limsup _{i \in I} M \omega\left(k_{i}, f, \mathrm{v}\right)+L=M \omega(k, f, \mathrm{v})+L .
\end{aligned}
$$

Fixemos $f_{0} \in C_{0}(K, X)$ tal que $\left\|f_{0}\right\|=\|\mathrm{v}\| / 2$ e $f_{0}(k)=\mathrm{v} / 2$, e segue de modo análogo ao feito em (3.7) que

$$
\liminf _{i \in I}\left\|T f_{0}\left(\varphi\left(k_{i}\right)\right)\right\| \geq\|\mathrm{v}\|\left(\frac{1}{M}-\frac{M}{2}\right)-2 L>0,
$$

onde a última desigualdade se deve a $\|\mathrm{v}\|>N^{\prime} \geq N_{0}$, conforme vimos em (3.8). Disso, pelo fato de $T f_{0}$ se anular no infinito, segue que $\left(\varphi\left(k_{i}\right)\right)_{i \in I}$ admite sub-rede convergente a um certo $s \in S$. Assim, podemos supor que $\varphi\left(k_{i}\right) \rightarrow s$ sem prejuízo da propriedade (3.16), da qual segue que para toda $f \in C_{0}(K, X)$,

$$
\|T f(s)-\mathrm{w}\| \leq M \omega(k, f, \mathrm{v})+L .
$$

Portanto, $s \in \Gamma_{\mathrm{w}}(k, \mathrm{v})$ e, como $\|\mathrm{v}\|>N^{\prime} \geq N, \Gamma_{\mathrm{w}}(k, \mathrm{v}) \subset \Phi_{N}(k)=\{\varphi(k)\}$, logo, $s=\varphi(k)$, concluindo assim a prova do Passo 1.

Pela versão simétrica do Passo 1, também segue que a função $\psi: S \rightarrow K$ é contínua. Assim, a conclusão de que $\varphi$ é um homeomorfismo decorre diretamente do próximo passo.

Passo 2. $\psi=\varphi^{-1}$.

Fixemos $k \in K$ e $\mathrm{v} \in X$ tal que $\|\mathrm{v}\|>M\left(N^{\prime}+L\right)$. Como $\|\mathrm{v}\|>M\left(N^{\prime}+L\right) \geq N^{\prime}$, segue do Lema 3.10 que existe $\mathrm{w} \in X$ com $\|\mathrm{w}\|=\|\mathrm{v}\| / M-L$ de modo que $\Gamma_{\mathrm{w}}(k, \mathrm{v})=\{\varphi(k)\}$. Mas note que

$$
\|\mathrm{w}\|=\|\mathrm{v}\| / M-L>M\left(N^{\prime}+L\right) / M-L=N^{\prime},
$$

logo, pela versão simétrica do Lema 3.10, existe $\mathrm{z} \in X$ tal que $\Lambda_{\mathrm{z}}(\varphi(k), \mathrm{w})=\{\psi(\varphi(k))\}$. Por outro 
lado, como $\|\mathrm{w}\|>N^{\prime} \geq N_{1}, \varphi(k) \in \Gamma_{\mathrm{w}}(k, \mathrm{v})$ e $\Lambda_{\mathrm{z}}(\varphi(k)) \neq \emptyset$, aplicando o lema da simetria (Lema $3.7)$, segue que $\Lambda_{\mathrm{z}}(\varphi(k), \mathrm{w})=\{k\}$. Portanto, $k=\psi(\varphi(k))$.

Deste modo, concluímos que $\psi \circ \varphi=I d_{K}$. De modo análogo, concluímos que $\varphi \circ \psi=I d_{S}$. Segue dessas duas conclusões que $\psi=\varphi^{-1}$. 


\section{Capítulo 4}

\section{Versão não-linear e vetorial para o Teorema de Amir-Cambern}

Neste capítulo, dedicamo-nos à prova do Teorema 1.16. Conforme dito anteriormente, faremos a prova através da verificação da existência de uma constante $N \geq M L$ satisfazendo a hipótese fundamental dos conjuntos de aproximação (Definição 3.8).

Doravante, teremos fixados os espaços localmetne compactos $K$ e $S$, o espaço de Banach real $X$ de dimensão finita maior ou igual que 2 e uma $(M, L)$-quasi-isometria $T: C_{0}(K, X) \rightarrow C_{0}(S, X)$ conforme no enuciado do Teorema 1.16. Como $M<\sqrt{\mathcal{S}(X)} \leq \sqrt{2}$ (veja o item (2) da Proposição 2.16), estamos aptos a utilizar a técnica dos conjuntos de aproximação, desenvolvida no capítulo 3. Portanto, de antemão, já fixamos constantes $N_{0}$ e $N_{1}$, dadas respectivamente pelos Lemas 3.6 e 3.7 .

A seguir, vemos uma observação que será útil na demonstração do próximo lema.

Observação 4.1. Para quaisquer números reais $a, b$ e c, temos a identidade a seguir

$$
\min \{\max \{a, b\}, \max \{a, c\}\}=\max \{a, \min \{b, c\}\} .
$$

De fato, por um lado,

$$
\begin{aligned}
b, c \geq \min \{b, c\} & \Rightarrow \max \{a, b\}, \max \{a, c\} \geq \max \{a, \min \{b, c\}\} \\
& \Rightarrow \min \{\max \{a, b\}, \max \{a, c\}\} \geq \max \{a, \min \{b, c\}\}
\end{aligned}
$$

e, por outro, assumindo, sem perda de generalidade, que $b=\min \{b, c\}$, temos

$$
\min \{\max \{a, b\}, \max \{a, c\}\} \leq \max \{a, b\}=\max \{a, \min \{b, c\}\} .
$$

Lema 4.2. Existe $N_{2} \geq 0$ de modo que, para todo $k \in K$ e $\mathrm{v}, \mathrm{v}^{\prime} \in X$, satisfazendo $\|\mathrm{v}\|,\left\|\mathrm{v}^{\prime}\right\|>N_{2}$ $e\left\|\mathrm{v}+\mathrm{v}^{\prime}\right\|<1$, se $s \in \Gamma_{\mathrm{w}}(k, \mathrm{v})$ e $s^{\prime} \in \Gamma_{\mathrm{w}^{\prime}}\left(k, \mathrm{v}^{\prime}\right)$, então $s=s^{\prime}$.

Demonstração. Suponha que $s \in \Gamma_{\mathrm{w}}(k, \mathrm{v}), s^{\prime} \in \Gamma_{\mathrm{w}^{\prime}}\left(k, \mathrm{v}^{\prime}\right)$ e $s \neq s^{\prime}$, e veremos que, para $N_{2}$ suficientemente grande, obtemos uma contradição.

Podemos supor, sem perda de generalidade, que $\left\|\mathrm{v}^{\prime}\right\| \geq\|\mathrm{v}\|$, e consequentemente, $\left\|\mathrm{w}^{\prime}\right\| \geq\|\mathrm{w}\|$. Como $s \neq s^{\prime}$, existe $g \in C_{0}(S, X)$ de modo que $\|g\|=\left\|\mathrm{w}^{\prime}\right\|, g(s)=-\mathrm{w}$ e $g\left(s^{\prime}\right)=-\mathrm{w}^{\prime}$ (veja Lema 2.15.(3)). Aplicando as definições de $s \in \Gamma_{\mathrm{w}}(k, \mathrm{v})$ e $s^{\prime} \in \Gamma_{\mathrm{w}^{\prime}}\left(k, \mathrm{v}^{\prime}\right)$ à função $T^{-1} g \in C_{0}(K, X)$, obtemos, respectivamente, que

$$
2\|\mathrm{w}\|=\|g(s)-\mathrm{w}\|=\left\|T\left(T^{-1} g\right)(s)-\mathrm{w}\right\| \leq M \omega\left(k, T^{-1} g, \mathrm{v}\right)+L,
$$

e

$$
2\left\|\mathrm{w}^{\prime}\right\|=\left\|g\left(s^{\prime}\right)-\mathrm{w}^{\prime}\right\|=\left\|T\left(T^{-1} g\right)\left(s^{\prime}\right)-\mathrm{w}^{\prime}\right\| \leq M \omega\left(k, T^{-1} g, \mathrm{v}^{\prime}\right)+L
$$


Como $\|\mathrm{w}\|=\|\mathrm{v}\| / M-L$, por (4.1), obtemos

$$
\frac{2\|\mathrm{v}\|}{M} \leq M \omega\left(k, T^{-1} g, \mathrm{v}\right)+3 L,
$$

e como $\left\|\mathrm{w}^{\prime}\right\|=\left\|\mathrm{v}^{\prime}\right\| / M-L \mathrm{e}\left\|\mathrm{v}+\mathrm{v}^{\prime}\right\|<1$, de acordo com (4.2), temos que

$$
\begin{aligned}
\frac{2\|\mathrm{v}\|}{M} & \leq 2\left(\frac{\left\|\mathrm{v}^{\prime}\right\|}{M}-L\right)+\frac{2\left\|\mathrm{v}^{\prime}+\mathrm{v}\right\|}{M}+2 L=2\left\|\mathrm{w}^{\prime}\right\|+\frac{2}{M}+2 L \\
& \leq M \omega\left(k, T^{-1} g, \mathrm{v}^{\prime}\right)+\frac{2}{M}+3 L
\end{aligned}
$$

e usando novamente $\left\|\mathrm{v}+\mathrm{v}^{\prime}\right\|<1$, vemos que

$$
\begin{aligned}
\omega\left(k, T^{-1} g, \mathrm{v}^{\prime}\right) & =\max \left\{\left\|T^{-1} g\right\|,\left\|T^{-1} g(k)-\mathrm{v}^{\prime}\right\|\right\} \\
& \leq \max \left\{\left\|T^{-1} g\right\|,\left\|T^{-1} g(k)+\mathrm{v}\right\|\right\}+1 \\
& =\omega\left(k, T^{-1} g,-\mathrm{v}\right)+1 .
\end{aligned}
$$

Portanto, por (4.4) deduzimos que

$$
\frac{2\|\mathrm{v}\|}{M} \leq M \omega\left(k, T^{-1} g,-\mathrm{v}\right)+M+\frac{2}{M}+3 L .
$$

Então, fazendo $\Delta=M+\frac{2}{M}+3 L$, segue de (4.3) e (4.5) que

$$
\frac{2\|\mathrm{v}\|}{M} \leq M \min \left\{\omega\left(k, T^{-1} g,-\mathrm{v}\right), \omega\left(k, T^{-1} g, \mathrm{v}\right)\right\}+\Delta .
$$

Isto é, $2\|\mathrm{v}\| / M$ é menor ou igual que

$$
M \min \left\{\max \left\{\left\|T^{-1} g\right\|,\left\|T^{-1} g(k)+\mathrm{v}\right\|\right\}, \max \left\{\left\|T^{-1} g\right\|,\left\|T^{-1} g(k)-\mathrm{v}\right\|\right\}\right\}+\Delta .
$$

Usando a identidade da Observação 4.1, com

$$
a=\left\|T^{-1} g\right\|, \quad b=\left\|T^{-1} g(k)+\mathrm{v}\right\| \text { and } c=\left\|T^{-1} g(k)-\mathrm{v}\right\|,
$$

concluímos que

$$
\frac{2\|\mathrm{v}\|}{M} \leq M \max \left\{\left\|T^{-1} g\right\|, \min \left\{\left\|T^{-1} g(k)+\mathrm{v}\right\|,\left\|T^{-1} g(k)-\mathrm{v}\right\|\right\}\right\}+\Delta .
$$

Então, aplicando o Lema 2.17 com $x=T^{-1} g(k)$ e $y=\mathrm{v}$, concluímos que

$$
\frac{2\|\mathrm{v}\|}{M} \leq M \max \left\{\left\|T^{-1} g\right\|, \frac{2}{\mathcal{S}(X)} \max \left\{\left\|T^{-1} g(k)\right\|,\|\mathrm{v}\|\right\}\right\}+\Delta,
$$

e lembrando que $\mathcal{S}(X) \leq 2$, obtemos

$$
\frac{2\|\mathrm{v}\|}{M} \leq \frac{2 M}{\mathcal{S}(X)} \max \left\{\left\|T^{-1} g\right\|,\|\mathrm{v}\|\right\}+\Delta .
$$

Por outro lado, fazendo $\Delta^{\prime}=1-(M-1) L$ concluímos que

$$
\left\|T^{-1} g\right\| \leq M\|g\|+L=M\left(\left\|\mathrm{v}^{\prime}\right\| / M-L\right)+L \leq\|\mathrm{v}\|+\left\|\mathrm{v}+\mathrm{v}^{\prime}\right\|-(M-1) L \leq\|\mathrm{v}\|+\Delta^{\prime} .
$$


Então, por (4.6) e (4.7), segue que

$$
\frac{2\|\mathrm{v}\|}{M} \leq \frac{2 M}{\mathcal{S}(X)}\left(\|\mathrm{v}\|+\Delta^{\prime}\right)+\Delta
$$

isto é,

$$
2\|\mathrm{v}\|\left(\frac{1}{M}-\frac{M}{\mathcal{S}(X)}\right) \leq \frac{2 M \Delta^{\prime}}{\mathcal{S}(X)}+\Delta
$$

Como $M^{2}<\mathcal{S}(X)$, temos que $1 / M-M / \mathcal{S}(X)>0$. Logo, existe $N_{2} \geq 0$, dependente apenas de $M, L$ e $\mathcal{S}(X)$, tal que a desigualdade acima é uma contradição sempre que $\|\mathrm{v}\|>N_{2}$.

No que segue, fixamos uma constante $N_{2}$ dada pelo Lema acima tal que $N_{2} \geq \max \left\{N_{0}, N_{1}\right\}$.

Corolário 4.3. Para todo $k \in K, s, s^{\prime} \in S$, e v, $\mathrm{v}^{\prime} \in X$, com $\|\mathrm{v}\|>N_{2},\left\|\mathrm{v}^{\prime}\right\|>N_{2}$ e $\left\|\mathrm{v}-\mathrm{v}^{\prime}\right\|<1$, se $s \in \Gamma_{\mathrm{w}}(k, \mathrm{v})$ e $s^{\prime} \in \Gamma_{\mathrm{w}^{\prime}}\left(k, \mathrm{v}^{\prime}\right)$ para certos $\mathrm{w}, \mathrm{w}^{\prime} \in X$, então $s=s^{\prime}$.

Demonstração. Como $\|-\mathrm{v}\|>N_{2} \geq N_{0}$, pelo Lema 3.6, existe $\mathrm{w}^{\prime \prime} \in X$ tal que $\Gamma_{\mathrm{w}^{\prime \prime}}(k,-\mathrm{v}) \neq \emptyset$. Tome $s^{\prime \prime} \in \Gamma_{\mathrm{w}^{\prime \prime}}(k,-\mathrm{v})$.

Observe que, como $s^{\prime \prime} \in \Gamma_{\mathrm{w}^{\prime \prime}}(k,-\mathrm{v}), s \in \Gamma_{\mathrm{w}}(k, \mathrm{v})$ e $\|\mathrm{v}+(-\mathrm{v})\|=0<1$, segue do Lema 4.2 que $s^{\prime \prime}=s$.

Além disso, como $s^{\prime \prime} \in \Gamma_{\mathrm{w}^{\prime \prime}}(k,-\mathrm{v}), s^{\prime} \in \Gamma_{\mathrm{w}^{\prime}}\left(k, \mathrm{v}^{\prime}\right)$ e $\left\|(-\mathrm{v})+\mathrm{v}^{\prime}\right\|<1$, novamente pelo Lema 4.2 concluímos que $s^{\prime \prime}=s^{\prime}$. Logo, $s=s^{\prime}$.

Proposição 4.4. A constante $N_{2}$ fixada acima satisfaz a hipótese fundamental.

Demonstração. Defina $A=\left\{\mathrm{v} \in X:\|\mathrm{v}\|>N_{2}\right\}$. Dado $k \in K$ para provarmos que o conjunto $\Phi_{N_{2}}(k)$ é unitário, basta verificarmos que

$$
\text { se } s \in \Gamma_{\mathrm{w}}(k, \mathrm{v}) \text { e } s^{\prime} \in \Gamma_{\mathrm{w}^{\prime}}\left(k, \mathrm{v}^{\prime}\right) \text { para certos } \mathrm{v}, \mathrm{v}^{\prime} \in A \text {, então } s=s^{\prime} .
$$

De fato, (4.8) implica que o conjunto $\Phi_{N_{2}}(k)$ tem no máximo um elemento. Por outro lado, lembrando que $N_{2} \geq N_{0}$, basta aplicar o lema da existência para algum $\mathrm{v} \in X$ com $\|\mathrm{v}\|>N_{2}$ para constatar que $\Phi_{N_{2}}(k)$ é não-vazio. Assim segue que $\Phi_{N_{2}}(k)$ é unitário. Por simetria dos resultados, conclui-se também que $\Psi_{N_{2}}(s)$ é unitário para todo $s \in S$.

Vamos à verificação de (4.8). Suponha que $s \in \Gamma_{\mathrm{w}}(k, \mathrm{v})$ e $s^{\prime} \in \Gamma_{\mathrm{w}^{\prime}}\left(k, \mathrm{v}^{\prime}\right)$ para certos v, $\mathrm{v}^{\prime} \in A$ e $\mathrm{w}, \mathrm{w}^{\prime} \in X$. Analisamos dois casos abaixo.

Caso 1. $\operatorname{dim} X \geq 2$.

Neste caso, $A$ é conexo por poligonais. Então, podemos encontrar pontos $\mathrm{u}_{0}, \ldots, \mathrm{u}_{n}$ em $A$, tais que $\mathrm{u}_{0}=\mathrm{v}^{\prime}, \mathrm{u}_{n}=\mathrm{v}$ e $\left\|\mathrm{u}_{j}-\mathrm{u}_{j-1}\right\|<1$ para todo $1 \leq j \leq n$. Faça $s_{0}=s^{\prime}$ e $s_{n}=s$. De acordo com o lema da existência, para cada $1 \leq j \leq n-1$, existe $s_{j} \in S$ e $\mathrm{w}_{j} \in X$ tal que $s_{j} \in \Gamma_{\mathrm{w}_{j}}\left(k, \mathrm{u}_{j}\right)$.

Para cada $1 \leq j \leq n$, como $\left\|\mathrm{u}_{j}-\mathrm{u}_{j-1}\right\|<1$, o Corolário 4.3 implica que $s_{j}=s_{j-1}$. Usando esse fato repetidas vezes, concluímos que $s^{\prime}=s_{1}=\ldots=s_{n-1}=s$.

Caso 2. $X=\mathbb{R}$. Neste caso, fixe $\mathrm{w}^{\prime \prime}$ tal que $\Gamma_{\mathrm{w}^{\prime \prime}}(k,-\mathrm{v}) \neq \emptyset$. Então, pelo Lema 4.2 , temos

$$
\Gamma_{\mathrm{w}^{\prime \prime}}(k,-\mathrm{v})=\Gamma_{\mathrm{w}}(k, \mathrm{v})=\{s\} .
$$

Como $A=\left(-\infty,-N_{2}\right) \cup\left(N_{2},+\infty\right)$, existe um caminho em $A$ conectando $\mathrm{v}^{\prime}$ a um dos pontos $\mathrm{v}$ ou $-\mathrm{v}$. A partir disso, basta procedermos de modo análogo ao Caso 1 para concluirmos que $s^{\prime}=s$. 



\section{Capítulo 5}

\section{Versão linear vetorial}

Neste capítulo, nos dedicaremos à prova do Teorema 1.24. No intuito de formalizar as propriedades geométricas essenciais para o resultado, definimos abaixo o conceito de constante de Cambern, que as sumariza.

Definição 5.1. Seja $X$ um espaço de Hilbert real de dimensão $n \geq 2$. Dizemos que $c>\sqrt{2}$ é uma constante de Cambern para $X$, se c satisfaz a desigualdade

$$
4 c^{2}+2 \sqrt{3} c \sqrt{c^{2}-2}<9
$$

e não existem $\mathrm{u}^{\prime}, \mathrm{u}_{1}, \ldots, \mathrm{u}_{n} \in X \quad e \mathrm{w}^{\prime}, \mathrm{w}_{1}, \ldots, \mathrm{w}_{n} \in S_{X}$ satisfazendo simultaneamente as seguintes desigualdades:

$$
\begin{aligned}
& \left(C_{1}\right)\left\|\mathrm{u}^{\prime} \pm\left(\mathrm{u}_{i}-\mathrm{w}_{i}\right)\right\| \leq c / 2, \forall 1 \leq i \leq n . \\
& \left(C_{2}\right)\left\|\mathrm{u}_{i} \pm\left(\mathrm{u}^{\prime}-\mathrm{w}^{\prime}\right)\right\| \leq c / 2, \forall 1 \leq i \leq n . \\
& \left(C_{3}\right)\left\|\sqrt{2} \mathrm{u}^{\prime}+\lambda \mathrm{u}_{i} \pm \theta \mathrm{u}_{j}\right\| \leq c / \sqrt{2}, \forall 1 \leq i, j \leq n, i \neq j \text { and } \forall \lambda, \theta \in\{-1,1\} .
\end{aligned}
$$

A prova ocorrerá em duas etapas. Primeiramente, na Seção 5.1, provaremos a existência de constantes de Cambern, por meio do lema abaixo.

Lema 5.2. Seja $X$ um espaço de Hilbert real de dimensão $n \geq 2$. Se $\delta>0$ satisfaz

$$
n-1<\frac{2-\delta}{10 \delta+8 \sqrt{2 \delta+\delta^{2}}}
$$

então $\sqrt{2+\delta}$ é uma constante de Cambern para $X$.

Posteriormente, na Seção 5.2, provaremos a seguinte versão do teorema de Cambern válido sempre que $M^{2}$ for uma constante de Cambern.

Teorema 5.3. Seja $X$ um espaço de Hilbert real de dimensão $n \geq 2$. Suponha que $M^{2}$ é uma constante de Cambern para $X$. Se $K$ e $S$ são espaços de Hausdorff localmente compactos e existe um isomorfismo $T$ de $C_{0}(K, X)$ sobre $C_{0}(S, X)$ satisfazendo

$$
\frac{\|f\|}{M} \leq\|T(f)\| \leq M\|f\|,
$$

para toda $f \in C_{0}(K, X)$, então $K$ e $S$ são homeomorfos.

A combinação dos resultados acima nos permite concluir a demonstração para o Teorema 1.24.

Demonstração do Teorema 1.24. Fixe $\delta>0$ satisfazendo as hipótesed do Lema 5.2 e suponha que $\|T\|\left\|T^{-1}\right\|<\sqrt{2+\delta}$. Faça $\tau=T\left\|T^{-1}\right\| /(2+\delta)^{1 / 4}$ e $M=\sqrt[4]{2+\delta}$. Então, $M^{2}=\sqrt{2+\delta}$ é uma 
constante de Cambern para $X$ e é simples verificar que $\tau$ é um isomorfismo de $C_{0}(K, X)$ sobre $C_{0}(S, X)$, satisfazendo

$$
\frac{\|f\|}{M} \leq\|\tau(f)\| \leq M\|f\|,
$$

para toda $f \in C_{0}(K, X)$. Então, pelo Teorema 5.3, segue o resultado.

A demonstração fará uso da técnica dos conjuntos de aproximação, desenvolvida no capítulo 3, desta vez aplicada para o contexto linear. Pela propriedade fundamental (Proposição 3.9), a demonstração se reduzirá a mostrar que existe um $N>0$ satisfazendo a hipótese fundamental (Definição 3.8).

Começamos estabelecendo algumas convenções. No decorrer deste capítulo, estarão fixos os espaços localmente compactos $K$ e $S$, o espaço de Hilbert real $X$ de dimensão finita maior ou igual que 2, um isomorfismo linear $T$ de $C_{0}(K, X)$ sobre $C_{0}(S, X)$ e $M$ satisfazendo a seguinte propriedade:

$$
\frac{1}{M}\|f\| \leq\|T f\| \leq M\|f\|, \forall f \in C_{0}(K, X) .
$$

Recordando a definição de $(M, L)$-quasi-isometria (Definição 1.4), vemos que a propriedade acima implica que $T$ é uma $(M, 0)$-quasi-isometria e, para estarmos aptos a utilizar a técnica dos conjuntos de aproximação, aqui supomos que $M<\sqrt{2}$.

A linearidade tem um papel essencial no desenvolvimento desse resultado pois adiciona boas propriedades aos conjuntos de aproximação, que sumarizamos abaixo.

Proposição 5.4. Sejam $k \in K e \mathrm{v} \in X \operatorname{com} \mathrm{v} \neq 0$. Então:

(1) Existe $\mathrm{w} \in X$ tal que $\Gamma_{\mathrm{w}}(k, \mathrm{v}) \neq \emptyset$.

(2) Suponha que $s \in \Gamma_{\mathrm{w}}(k, \mathrm{v})$ e que $\Lambda_{\mathrm{z}}(s, \mathrm{w}) \neq \emptyset$. Então $\Lambda_{\mathrm{z}}(s, \mathrm{w})=\{k\}$.

(3) Para todo $t \in \mathbb{R}$ com $t \neq 0$, temos que $\Gamma_{\mathrm{w}}(k, \mathrm{v})=\Gamma_{t \mathrm{w}}(k, t \mathrm{v})$.

Demonstração. Conforme discutimos acima, $T$ é uma $(M, 0)$-quasi-isometria. O fato da constante $L$ ser nula implica que as constantes $N_{0}$ e $N_{1}$, que nos permitem utilizar os Lemas 3.6 e 3.7, podem ser ambas tomadas iguais a 0 . Como consequência, ambos os lemas são válidos para qualquer $\mathrm{v} \neq 0$, donde segue imediatamente os itens (1) e (2).

Para demonstrar o item (3), será suficiente verificar que

$$
\Gamma_{\mathrm{w}}(k, \mathrm{v}) \subset \Gamma_{t \mathrm{w}}(k, t \mathrm{v}) .
$$

De fato, uma vez isso provado, como v, w e $t$ são arbitrários, podemos aplicar $(5.2)$ para $\Gamma_{t \mathrm{w}}(k, t \mathrm{v})$ e $1 / t$, e assim obter o outro sentido da continência:

$$
\Gamma_{t \mathrm{w}}(k, t \mathrm{v}) \subset \Gamma_{\frac{1}{t} t \mathrm{w}}\left(k, \frac{1}{t} t \mathrm{v}\right)=\Gamma_{\mathrm{w}}(k, \mathrm{v}) .
$$

Vamos à verificação de (5.2). Fixe $s \in \Gamma_{\mathrm{w}}(k, \mathrm{v})$ e vejamos a seguir que $s \in \Gamma_{t \mathrm{w}}(k, t \mathrm{v})$. Dada $f \in C_{0}(K, X)$ tome $f^{\prime}=t^{-1} f$. Pela definição de $\Gamma_{\mathrm{w}}(k, \mathrm{v})$ segue que

$$
\left\|T f^{\prime}(s)-\mathrm{w}\right\| \leq M \omega\left(k, f^{\prime}, \mathrm{v}\right),
$$

e então

$$
\|T f(s)-t \mathrm{w}\|=|t|\left\|T f^{\prime}(s)-\mathrm{w}\right\| \leq M|t| \omega\left(k, f^{\prime}, \mathrm{v}\right)=M \omega(k, f, t \mathrm{v}) .
$$

Consequentemente, $s \in \Gamma_{t \mathrm{w}}(k, t \mathrm{v})$, como queríamos. 


\subsection{Sobre a existência de constantes de Cambern}

Nesta seção, apresentamos a prova do Lema 5.2. A seguir, denotaremos por $\langle\cdot, \cdot\rangle$ o produto interno de $X$. Também faremos uso do seguinte caso particular da desigualdade de Hölder para somatórios finitos (veja [Rud87, p. 63]):

$$
\left|\sum_{i=1}^{k} a_{i}\right| \leq \sqrt{k}\left(\sum_{i=1}^{k} a_{i}^{2}\right)^{\frac{1}{2}}, \forall a_{1}, \ldots, a_{k} \in \mathbb{R} .
$$

Lema 5.5. Seja $c=\sqrt{2+\delta} \operatorname{com} \delta>0$. Suponha que existam $\mathrm{u}^{\prime}, \mathrm{u}_{1}, \ldots, \mathrm{u}_{n} \in X$ e $\mathrm{w}^{\prime}, \mathrm{w}_{1}, \ldots, \mathrm{w}_{n} \in$ $S_{X}$ satisfazendo simultaneamente as desigualdades $\left(C_{1}\right),\left(C_{2}\right)$ e $\left(C_{3}\right)$ da definição de constante de Cambern para X. Então,

$\left(D_{1}\right)\left|\left\langle\mathrm{w}^{\prime}, \mathrm{w}_{i}\right\rangle\right| \leq \delta / 2, \forall 1 \leq i \leq n$.

$\left(D_{2}\right)\left\|\mathrm{u}^{\prime} \pm \mathrm{u}_{i}-\left(\mathrm{w}^{\prime} \pm \mathrm{w}_{i}\right) / 2\right\|^{2} \leq \delta / 2, \forall 1 \leq i \leq n$

$\left(D_{3}\right)$ Pondo $\mathrm{z}^{\prime}=\mathrm{u}^{\prime}-\mathrm{w}^{\prime} / 2$ e $\mathrm{z}_{i}=\mathrm{u}_{i}-\mathrm{w}_{i} / 2$, para todo $1 \leq i \leq n$, temos

$$
\sum_{\lambda, \theta \in\{-1,1\}}\left\|\sqrt{2} \mathrm{z}^{\prime}+\lambda \mathrm{z}_{i}+\theta \mathrm{z}_{j}\right\|^{2} \leq 4 \delta, \forall i \neq j
$$

$\left(D_{4}\right)\left|\left\langle\mathrm{w}_{i}, \mathrm{w}_{j}\right\rangle\right| \leq 5 \delta+\sqrt{32 \delta+16 \delta^{2}}, \forall i \neq j$.

Demonstração. $\left(D_{1}\right)$ Dados $1 \leq i \leq n$, pelas desigualdades de $\left(C_{1}\right)$ e $\left(C_{2}\right)$ com sinal '+', sabemos que

$$
\left\|\mathrm{u}^{\prime}+\mathrm{u}_{i}-\mathrm{w}_{i}\right\| \leq c / 2 \text { e }\left\|\mathrm{u}^{\prime}+\mathrm{u}_{i}-\mathrm{w}^{\prime}\right\| \leq c / 2
$$

Portanto,

$$
\left\|\mathrm{w}^{\prime}-\mathrm{w}_{i}\right\| \leq c,
$$

e pelas desigualdades de $\left(C_{1}\right)$ e $\left(C_{2}\right)$ com sinal '-', também sabemos que

$$
\left\|\mathrm{u}^{\prime}-\mathrm{u}_{i}+\mathrm{w}_{i}\right\| \leq c / 2 \text { e }\left\|\mathrm{u}^{\prime}-\mathrm{u}_{i}-\mathrm{w}^{\prime}\right\| \leq c / 2 .
$$

Portanto,

$$
\left\|\mathrm{w}^{\prime}+\mathrm{w}_{i}\right\| \leq c
$$

Consequentemente,

$$
2+2\left|\left\langle\mathrm{w}^{\prime}, \mathrm{w}_{i}\right\rangle\right|=\max \left\{\left\|\mathrm{w}^{\prime}+\mathrm{w}_{i}\right\|^{2},\left\|\mathrm{w}^{\prime}-\mathrm{w}_{i}\right\|^{2}\right\} \leq c^{2}=2+\delta,
$$

isto é, $\left|\left\langle\mathrm{w}^{\prime}, \mathrm{w}_{i}\right\rangle\right| \leq \delta / 2$.

$\left(D_{2}\right)$ Fixe $1 \leq i \leq n$. Como $\left\|\mathrm{w}^{\prime}\right\|=\left\|\mathrm{w}_{i}\right\|$, segue que $\mathrm{w}^{\prime}+\mathrm{w}_{i}$ é ortogonal a $\mathrm{w}^{\prime}-\mathrm{w}_{i}$. Tome $a, b \in \mathbb{R}$ $\mathrm{e} \mathrm{z} \in\left[\mathrm{w}^{\prime}+\mathrm{w}_{i}, \mathrm{w}^{\prime}-\mathrm{w}_{i}\right]^{\perp}$ tal que

$$
\mathrm{u}^{\prime}+\mathrm{u}_{i}=a\left(\mathrm{w}^{\prime}+\mathrm{w}_{i}\right)+b\left(\mathrm{w}^{\prime}-\mathrm{w}_{i}\right)+\mathrm{z} .
$$

A desigualdade de $\left(C_{1}\right)$ com sinal '+' implica que

$$
\left\|\mathrm{u}^{\prime}+\mathrm{u}_{i}-\mathrm{w}_{i}\right\|^{2} \leq c^{2} / 4 .
$$

Assim,

$$
\left\|\left[a\left(\mathrm{w}^{\prime}+\mathrm{w}_{i}\right)+b\left(\mathrm{w}^{\prime}-\mathrm{w}_{i}\right)+\mathrm{z}\right]-\left[\left(\mathrm{w}^{\prime}+\mathrm{w}_{i}\right) / 2-\left(\mathrm{w}^{\prime}-\mathrm{w}_{i}\right) / 2\right]\right\|^{2} \leq c^{2} / 4,
$$


isto é,

$$
\left\|(a-1 / 2)\left(\mathrm{w}^{\prime}+\mathrm{w}_{i}\right)+(b+1 / 2)\left(\mathrm{w}^{\prime}-\mathrm{w}_{i}\right)+\mathrm{z}\right\|^{2} \leq c^{2} / 4,
$$

e, portanto,

$$
(a-1 / 2)^{2}\left\|\mathrm{w}^{\prime}+\mathrm{w}_{i}\right\|^{2}+(b+1 / 2)^{2}\left\|\mathrm{w}^{\prime}-\mathrm{w}_{i}\right\|^{2}+\|\mathrm{z}\|^{2} \leq c^{2} / 4 .
$$

Por outro lado, pela desigualdade de $\left(C_{2}\right)$ com sinal ' + ', inferimos que

$$
\left\|\mathrm{u}^{\prime}+\mathrm{u}_{i}-\mathrm{w}^{\prime}\right\|^{2} \leq c^{2} / 4,
$$

isto é,

$$
(a-1 / 2)^{2}\left\|\mathrm{w}^{\prime}+\mathrm{w}_{i}\right\|^{2}+(b-1 / 2)^{2}\left\|\mathrm{w}^{\prime}-\mathrm{w}_{i}\right\|^{2}+\|\mathrm{z}\|^{2} \leq c^{2} / 4 .
$$

Então, de acordo com (5.6) e (5.7), deduzimos que

$$
(a-1 / 2)^{2}\left\|\mathrm{w}^{\prime}+\mathrm{w}_{i}\right\|^{2}+(|b|+1 / 2)^{2}\left\|\mathrm{w}^{\prime}-\mathrm{w}_{i}\right\|^{2}+\|\mathrm{z}\|^{2} \leq c^{2} / 4,
$$

ou equivalentemente,

$$
(a-1 / 2)^{2}\left\|\mathrm{w}^{\prime}+\mathrm{w}_{i}\right\|^{2}+b^{2}\left\|\mathrm{w}^{\prime}-\mathrm{w}_{i}\right\|^{2}+\|\mathrm{z}\|^{2} \leq c^{2} / 4-(1 / 4+|b|)\left\|\mathrm{w}^{\prime}-\mathrm{w}_{i}\right\|^{2} .
$$

Em seguida, fazendo uso da desigualdade de $\left(D_{1}\right)$, concluimos que

$$
\begin{aligned}
\left\|\mathrm{u}^{\prime}+\mathrm{u}_{i}-\left(\mathrm{w}^{\prime}+\mathrm{w}_{i}\right) / 2\right\|^{2} & =\left\|(a-1 / 2)\left(\mathrm{w}^{\prime}+\mathrm{w}_{i}\right)+b\left(\mathrm{w}^{\prime}-\mathrm{w}_{i}\right)+\mathrm{z}\right\|^{2} \\
& =(a-1 / 2)^{2}\left\|\mathrm{w}^{\prime}+\mathrm{w}_{i}\right\|^{2}+b^{2}\left\|\mathrm{w}^{\prime}-\mathrm{w}_{i}\right\|^{2}+\|\mathrm{z}\|^{2} \\
& \leq c^{2} / 4-(1 / 4+|b|)\left\|\mathrm{w}^{\prime}-\mathrm{w}_{i}\right\|^{2} \\
& \leq c^{2} / 4-\left\|\mathrm{w}^{\prime}-\mathrm{w}_{i}\right\|^{2} / 4 \\
& =c^{2} / 4-\left(2-2\left\langle\mathrm{w}^{\prime}, \mathrm{w}_{i}\right\rangle\right) / 4 \\
& \leq c^{2} / 4-(2-\delta) / 4=(2+\delta) / 4-(2-\delta) / 4=\delta / 2 .
\end{aligned}
$$

Analogamente, tomando $a, b \in \mathbb{R}$ e $\mathrm{z} \in\left[\mathrm{w}^{\prime}+\mathrm{w}_{i}, \mathrm{w}^{\prime}-\mathrm{w}_{i}\right]^{\perp}$ tal que

$$
u^{\prime}-u_{i}=a\left(\mathrm{w}^{\prime}+\mathrm{w}_{i}\right)+b\left(\mathrm{w}^{\prime}-\mathrm{w}_{i}\right)+\mathrm{z},
$$

partindo desigualdade de $\left(C_{1}\right)$ com sinal '-', seguindo procedimento análogo ao feito para obtermos (5.6), vemos que

$$
(a+1 / 2)^{2}\left\|\mathrm{w}^{\prime}+\mathrm{w}_{i}\right\|^{2}+(b-1 / 2)^{2}\left\|\mathrm{w}^{\prime}-\mathrm{w}_{i}\right\|^{2}+\|\mathrm{z}\|^{2} \leq c^{2} / 4 .
$$

Por sua vez, partindo da desigualdade de $\left(C_{2}\right)$ com o sinal '-', seguindo procedimento análogo ao que nos levou a (5.7), obtemos

$$
(a-1 / 2)^{2}\left\|\mathrm{w}^{\prime}+\mathrm{w}_{i}\right\|^{2}+(b-1 / 2)^{2}\left\|\mathrm{w}^{\prime}-\mathrm{w}_{i}\right\|^{2}+\|\mathrm{z}\|^{2} \leq c^{2} / 4 .
$$

Portanto, segue a versão análoga de (5.8)

$$
(|a|+1 / 2)^{2}\left\|\mathrm{w}^{\prime}+\mathrm{w}_{i}\right\|^{2}+(b-1 / 2)^{2}\left\|\mathrm{w}^{\prime}-\mathrm{w}_{i}\right\|^{2}+\|\mathrm{z}\|^{2} \leq c^{2} / 4,
$$

e desenvolvendo essa desigualdade como no caso anterior, obtemos

$$
\left\|\mathrm{u}^{\prime}-\mathrm{u}_{i}-\left(\mathrm{w}^{\prime}-\mathrm{w}_{1}\right) / 2\right\|^{2} \leq \delta / 2 .
$$

$\left(D_{3}\right)$ Fazendo uso das duas desigualdades de $\left(D_{2}\right)$ e da regra do paralelogramo, obtemos que

$$
\left\|\mathrm{z}^{\prime}\right\|^{2}+\left\|\mathrm{z}_{i}\right\|^{2}=\left\|\mathrm{u}^{\prime}-\mathrm{w}^{\prime} / 2\right\|^{2}+\left\|\mathrm{u}_{i}-\mathrm{w}_{i} / 2\right\|^{2} \leq \delta / 2, \forall 1 \leq i \leq n .
$$


Então, dados $1 \leq i, j \leq n$, com $i \neq j$, por (5.9), aplicando duas vezes a regra do paralelogramo, obtemos

$$
\begin{aligned}
\sum_{\lambda, \theta \in\{-1,1\}}\left\|\sqrt{2} \mathrm{z}^{\prime}+\lambda \mathrm{z}_{i}+\theta \mathrm{z}_{j}\right\|^{2} & =4\left\|\sqrt{2} \mathrm{z}^{\prime}\right\|^{2}+4\left\|\mathrm{z}_{i}\right\|^{2}+4\left\|\mathrm{z}_{j}\right\|^{2} \\
& =4\left(\left\|\mathrm{z}^{\prime}\right\|^{2}+\left\|\mathrm{z}_{i}\right\|^{2}\right)+4\left(\left\|\mathrm{z}^{\prime}\right\|^{2}+\left\|\mathrm{z}_{j}\right\|^{2}\right) \\
& \leq 4 \delta / 2+4 \delta / 2=4 \delta .
\end{aligned}
$$

$\left(D_{4}\right)$ Fixe $\lambda \in\{-1,1\}$. As desigualdades de $\left(C_{3}\right)$ implicam que

$$
\begin{aligned}
\left\|\sqrt{2} \mathrm{w}^{\prime} / 2 \pm\left(\mathrm{w}_{i} / 2+\lambda \mathrm{w}_{j} / 2\right)\right\| & \leq\left\|\sqrt{2} \mathrm{u}^{\prime} \pm\left(\mathrm{u}_{i}+\lambda \mathrm{u}_{j}\right)\right\|+\left\|\sqrt{2} \mathrm{z}^{\prime} \pm\left(\mathrm{z}_{i}+\lambda \mathrm{z}_{j}\right)\right\| \\
& \leq c / \sqrt{2}+\left\|\sqrt{2} \mathrm{z}^{\prime} \pm\left(\mathrm{z}_{i}+\lambda \mathrm{z}_{j}\right)\right\|,
\end{aligned}
$$

e, portanto,

$$
\left\|\sqrt{2} \mathrm{w}^{\prime} / 2 \pm\left(\mathrm{w}_{i} / 2+\lambda \mathrm{w}_{j} / 2\right)\right\|^{2} \leq c^{2} / 2+\sqrt{2} c\left\|\sqrt{2} \mathrm{z}^{\prime} \pm\left(\mathrm{z}_{i}+\lambda \mathrm{z}_{j}\right)\right\|+\left\|\sqrt{2} \mathrm{z}^{\prime} \pm\left(\mathrm{z}_{i}+\lambda \mathrm{z}_{j}\right)\right\|^{2},
$$

e, então, pondo

$$
\Delta=2\left\|\sqrt{2} \mathrm{w}^{\prime} / 2\right\|^{2}+2\left\|\mathrm{w}_{i} / 2+\lambda \mathrm{w}_{j} / 2\right\|^{2},
$$

pela regra do paralelogramo, vemos que

$$
\begin{gathered}
\Delta=\left\|\sqrt{2} \mathrm{w}^{\prime} / 2+\left(\mathrm{w}_{i} / 2+\lambda \mathrm{w}_{j} / 2\right)\right\|^{2}+\left\|\sqrt{2} \mathrm{w}^{\prime} / 2-\left(\mathrm{w}_{i} / 2+\lambda \mathrm{w}_{j} / 2\right)\right\|^{2} \\
\leq c^{2}+\sqrt{2} c\left(\left\|\sqrt{2} \mathrm{z}^{\prime}+\left(\mathrm{z}_{i}+\lambda \mathrm{z}_{j}\right)\right\|+\left\|\sqrt{2} \mathrm{z}^{\prime}-\left(\mathrm{z}_{i}+\lambda \mathrm{z}_{j}\right)\right\|\right) \\
+\left(\left\|\sqrt{2} \mathrm{z}^{\prime}+\left(\mathrm{z}_{i}+\lambda \mathrm{z}_{j}\right)\right\|^{2}+\left\|\sqrt{2} \mathrm{z}^{\prime}-\left(\mathrm{z}_{i}+\lambda \mathrm{z}_{j}\right)\right\|^{2}\right) .
\end{gathered}
$$

Por outro lado, por (5.3) e pelas desigualdades de $\left(D_{3}\right)$, obtemos

$$
\left\|\sqrt{2} \mathrm{z}^{\prime}+\left(\mathrm{z}_{i}+\lambda \mathrm{z}_{j}\right)\right\|+\left\|\sqrt{2} \mathrm{z}^{\prime}-\left(\mathrm{z}_{i}+\lambda \mathrm{z}_{j}\right)\right\| \leq 2 \sqrt{2} \sqrt{\delta},
$$

$\mathrm{e}$

$$
\left\|\sqrt{2} \mathrm{z}^{\prime}+\left(\mathrm{z}_{i}+\lambda \mathrm{z}_{j}\right)\right\|^{2}+\left\|\sqrt{2} \mathrm{z}^{\prime}-\left(\mathrm{z}_{i}+\lambda \mathrm{z}_{j}\right)\right\|^{2} \leq 4 \delta .
$$

Então,

$$
2\left\|\sqrt{2} \mathrm{w}^{\prime} / 2\right\|^{2}+2\left\|\mathrm{w}_{i} / 2+\lambda \mathrm{w}_{j} / 2\right\|^{2}=\Delta \leq c^{2}+4 \sqrt{\delta} c+4 \delta .
$$

Usando que $\left\|\mathrm{w}_{i}+\lambda \mathrm{w}_{j}\right\|^{2}=2+2 \lambda\left\langle\mathrm{w}_{i}, \mathrm{w}_{j}\right\rangle$ e $c=\sqrt{2+\delta}$, concluímos que

$$
\begin{aligned}
2+\lambda\left\langle\mathrm{w}_{i}, \mathrm{w}_{j}\right\rangle & =2\left\|\sqrt{2} \mathrm{w}^{\prime} / 2\right\|^{2}+2\left\|\mathrm{w}_{i} / 2+\lambda \mathrm{w}_{j} / 2\right\|^{2} \\
& \leq c^{2}+4 \sqrt{\delta} c+4 \delta \\
& =2+\delta+4 \sqrt{\delta} \sqrt{2+\delta}+4 \delta,
\end{aligned}
$$

isto é,

$$
\lambda\left\langle\mathrm{w}_{i}, \mathrm{w}_{j}\right\rangle \leq 5 \delta+4 \sqrt{\delta} \sqrt{2+\delta} .
$$

Como $\lambda \in\{-1,1\}$ é arbitrário, o resultado segue.

Agora estamos aptos a provar o Lema 5.2.

Demonstração do Lema 5.2. Seja $c=\sqrt{2+\delta}$ para algum $\delta>0$ satisfazendo

$$
n-1<\frac{2-\delta}{10 \delta+8 \sqrt{2 \delta+\delta^{2}}}
$$


então,

$$
\begin{aligned}
4 c^{2}+2 \sqrt{3} c \sqrt{c^{2}-2} & =8+4 \delta+2 \sqrt{3} \sqrt{2 \delta+\delta^{2}}<8+5 \delta+4 \sqrt{2 \delta+\delta^{2}} \\
& <8+\frac{2-\delta}{2(n-1)} \leq 8+\frac{2-\delta}{2}<9 .
\end{aligned}
$$

Assuma por contradição que $c$ não é uma constante de Cambern para $X$. Então existem $\mathrm{u}^{\prime}, \mathrm{u}_{1}, \ldots, \mathrm{u}_{n} \in$ $X \mathrm{e} \mathrm{w}^{\prime}, \mathrm{w}_{1}, \ldots, \mathrm{w}_{n} \in S_{X}$ satisfazendo simultaneamente as desigualdades $\left(C_{1}\right),\left(C_{2}\right)$ e $\left(C_{3}\right)$ da Definição 5.1. Para propósitos de notação, faça $\mathrm{w}_{0}=\mathrm{w}^{\prime}$ e considere a matriz

$$
A=\left[\left\langle\mathrm{w}_{i-1}, \mathrm{w}_{j-1}\right\rangle\right]_{1 \leq i, j \leq n+1} .
$$

Provamos a seguir que, sob as condições atuais, a matriz $A$ é invertível. Disso segue que o conjunto $\left\{\mathrm{w}_{0}, \mathrm{w}_{1}, \ldots, \mathrm{w}_{n}\right\}$ é linearmente independente, nos dando uma contradição, uma vez que a dimensão de $X$ é $n$.

Para ver que $A$ é inversível basta verificarmos que para qualquer v $=\left(x_{0}, \ldots, x_{n}\right) \in \mathbb{R}^{n+1}$ nãonulo, temos $A \mathrm{v} \neq 0$. Escreva $A \mathrm{v}=\left(y_{0}, \ldots, y_{n}\right)$. Distinguimos dois casos abaixo:

Caso 1. $\left|x_{j}\right|=\max \left\{\left|x_{0}\right|, \ldots,\left|x_{n}\right|\right\}$ para algum $j \neq 0$. Assim, temos que

$$
\begin{aligned}
\left|y_{j}\right| & =\left|x_{0}\left\langle\mathrm{w}_{j}, \mathrm{w}_{0}\right\rangle+x_{1}\left\langle\mathrm{w}_{j}, \mathrm{w}_{1}\right\rangle+\ldots+x_{j}\left\langle\mathrm{w}_{j}, \mathrm{w}_{j}\right\rangle+\ldots+x_{n}\left\langle\mathrm{w}_{j}, \mathrm{w}_{n}\right\rangle\right| \\
& \geq\left|x_{j}\right||| \mathrm{w}_{j} \|^{2}-\sum_{\substack{l=0 \\
l \neq j}}^{n}\left|x_{l}\right|\left|\left\langle\mathrm{w}_{j}, \mathrm{w}_{l}\right\rangle\right| \geq\left|x_{j}\right|-\left|x_{j}\right| \sum_{\substack{l=0 \\
l \neq j}}^{n}\left|\left\langle\mathrm{w}_{j}, \mathrm{w}_{l}\right\rangle\right| \\
& =\left|x_{j}\right|\left(1-\left|\left\langle\mathrm{w}_{j}, \mathrm{w}_{0}\right\rangle\right|-\sum_{\substack{l=1 \\
l \neq j}}^{n}\left|\left\langle\mathrm{w}_{j}, \mathrm{w}_{l}\right\rangle\right|\right)
\end{aligned}
$$

Portanto, de acordo com as desigualdades $\left(D_{1}\right)$ e $\left(D_{4}\right)$ do Lema 5.5, deduzimos que

$$
\left|y_{j}\right| \geq\left|x_{j}\right|(1-\delta / 2-(n-1)(5 \delta+4 \sqrt{\delta} \sqrt{2+\delta}))>0 .
$$

Caso 2. $\left|x_{0}\right|=\max \left\{\left|x_{0}\right|, \ldots,\left|x_{n}\right|\right\}$. Nesse caso, novamente pelas desigualdades $\left(D_{1}\right)$ e $\left(D_{4}\right)$ dos Lemas 5.5, concluímos que

$$
\begin{aligned}
\left|y_{0}\right| & =\left|x_{0}\left\langle\mathrm{w}_{0}, \mathrm{w}_{0}\right\rangle+x_{1}\left\langle\mathrm{w}_{0}, \mathrm{w}_{1}\right\rangle+\ldots+x_{n}\left\langle\mathrm{w}_{0}, \mathrm{w}_{n}\right\rangle\right| \\
& \geq\left|x_{0}\right|\left\|\mathrm{w}_{0}\right\|^{2}-\sum_{l=1}^{n}\left|x_{l}\right|\left|\left\langle\mathrm{w}_{0}, \mathrm{w}_{l}\right\rangle\right| \geq\left|x_{0}\right|-\left|x_{0}\right| \sum_{l=1}^{n}\left|\left\langle\mathrm{w}_{0}, \mathrm{w}_{l}\right\rangle\right| \\
& \geq\left|x_{0}\right|(1-n \delta / 2)>0
\end{aligned}
$$

onde a última desigualdade acima decorre da contrapositiva do seguinte:

$$
\delta \geq \frac{n}{2} \Rightarrow\left(10 \delta+8 \sqrt{2 \delta+\delta^{2}}\right)(n-1) \geq \frac{20(n-1)}{n} \geq 10>2-\frac{2}{n} \geq 2-\delta .
$$

\subsection{Uma versão linear e vetorial para constantes de Cambern}

Nesta seção nos dedicamos a provar o Teorema 5.3. Portanto, em todos os resultados dessa seção estamos considerando que $M^{2}$ é uma constante de Cambern para o espaço $X$ fixado inicialmente. Tendo em vista a propriedade fundamental dos conjuntos de aproximação (Proposição 3.9), basta 
provar que, com essa hipótese sobre $M$, existe $N \geq 0$ satisfazendo a hipótese fundamental.

Antes de prosseguirmos, é importante discutirmos as definições dos conjuntos $\Phi_{N}(k)$ e $\Psi_{N}(s)$ no contexto linear (veja Definição 3.8). Vejamos que, devido à propriedade (3) da Proposição 5.4, temos que os conjuntos $\Phi_{N}(k)$ e $\Psi_{N}(s)$ independem da constante $N$. De fato, dados $0 \leq N<N^{\prime}$, fazendo $\alpha=N^{\prime} / N$, segue da propriedade citada que

$$
\begin{aligned}
\Phi_{N}(k) & =\bigcup\left\{\Gamma_{\mathrm{w}}(k, \mathrm{v}):\|\mathrm{v}\|>N \text { e }\|\mathrm{w}\|=\|\mathrm{v}\| / M\right\} \\
& =\bigcup\left\{\Gamma_{\alpha \mathrm{w}}(k, \alpha \mathrm{v}):\|\mathrm{v}\|>N \text { e }\|\mathrm{w}\|=\|\mathrm{v}\| / M\right\} \\
& =\bigcup\left\{\Gamma_{\mathrm{w}}(k, \mathrm{v}):\|\mathrm{v}\|>\alpha N \text { e }\|\mathrm{w}\|=\|\mathrm{v}\| / M\right\} \\
& =\bigcup\left\{\Gamma_{\mathrm{w}}(k, \mathrm{v}):\|\mathrm{v}\|>N^{\prime} \mathrm{e}\|\mathrm{w}\|=\|\mathrm{v}\| / M\right\} \\
& =\Phi_{N^{\prime}}(k) .
\end{aligned}
$$

Portanto, no que segue, estes conjuntos serão denotados sem o subíndice $N$. Neste cenário, provar que existe $N \geq 0$ satisfazendo a hipótese fundamental é equivalente a mostrar que todos os conjuntos $\Phi(k)$ e $\Psi(s)$ são unitários, que corresponde à Proposição 5.11. Antes de provar esta proposição, temos a seguir uma série de resultados intermediários.

Proposição 5.6. A cardinalidade de $\Phi(k)$ é no máximo 2, para todo $k \in K$.

Demonstração. Suponha que existem $s_{1}, s_{2}, s_{3} \in \Phi(k)$. Fazendo uso das propriedades (1) e (2) da Proposição 5.4, é simples concluir, para cada $1 \leq i \leq 3$, que $k \in \Psi\left(s_{i}\right)$, logo,

$$
k \in \Psi\left(s_{1}\right) \cap \Psi\left(s_{2}\right) \cap \Psi\left(s_{3}\right) .
$$

Pelo item (3) da Proposiçao 5.4, podemos tomar $\mathrm{w}_{1}, \mathrm{w}_{2}, \mathrm{w}_{3} \in X$ com norma $M$ e $\mathrm{z}_{1}, \mathrm{z}_{2}, \mathrm{z}_{3}$ satisfazendo

$$
k \in \Lambda_{\mathrm{z}_{1}}\left(s_{1}, \mathrm{w}_{1}\right) \cap \Lambda_{\mathrm{z}_{2}}\left(s_{2}, \mathrm{w}_{2}\right) \cap \Lambda_{\mathrm{z}_{3}}\left(s_{3}, \mathrm{w}_{3}\right) .
$$

Temos que $\left\|\mathrm{z}_{1}\right\|=\left\|\mathrm{z}_{2}\right\|=\left\|\mathrm{z}_{3}\right\|=1$. Fazendo uso do Lema 2.15(2), fixe $g_{1}, g_{2}, g_{3} \in C_{0}(K, X)$ tais que

(a) $\left\|g_{i}\right\|=M / 2$ para todo $1 \leq i \leq 3$.

(b) $g_{i}\left(s_{i}\right)=\mathrm{w}_{i} / 2$ para todo $1 \leq i \leq 3$.

(c) Os suportes de $g_{i}$ e $g_{j}$ sao disjuntos para todos $i \neq j$.

Defina $u_{i}=T^{-1} g_{i}(k)$ para cada $1 \leq i \leq 3$. Dados $1 \leq i, j \leq 3$ distintos, por conta de (c), temos que $\left\|g_{i} \pm g_{j}\right\|=M / 2$. Além disso, pelas propriedades (b) e (c),

$$
\left\|g_{i}\left(s_{j}\right) \pm g_{j}\left(s_{j}\right)-\left( \pm \mathrm{w}_{j}\right)\right\|=\left\|0 \pm \mathrm{w}_{j} / 2-\left( \pm \mathrm{w}_{j}\right)\right\|=\left\|\mathrm{w}_{j}\right\| / 2=M / 2 .
$$

Então,

$$
\omega\left(s_{j}, g_{i} \pm g_{j}, \pm \mathrm{w}_{j}\right)=\max \left\{\left\|g_{i} \pm g_{j}\right\|,\left\|g_{i}\left(s_{j}\right) \pm g_{j}\left(s_{j}\right)-\left( \pm \mathrm{w}_{j}\right)\right\|\right\}=M / 2 .
$$

Portanto, aplicando a definição de $k \in \Lambda_{\mathrm{z}_{j}}\left(s_{j}, \mathrm{w}_{j}\right)$ para $g_{i} \pm g_{j}$, obtemos

$$
\begin{aligned}
\left\|\mathrm{u}_{i} \pm\left(\mathrm{u}_{j}-\mathrm{z}_{j}\right)\right\| & =\left\|\mathrm{u}_{i} \pm \mathrm{u}_{j}-\left( \pm \mathrm{z}_{j}\right)\right\|=\left\|T^{-1}\left(g_{i} \pm g_{j}\right)(k)-\left( \pm \mathrm{z}_{j}\right)\right\| \\
& \leq M \omega\left(s_{j}, g_{i} \pm g_{j}, \pm \mathrm{w}_{j}\right)=\frac{M^{2}}{2}, \quad \forall i \neq j .
\end{aligned}
$$

Além disso, por (a) e (c), sabemos que

$$
\left\|g_{1}+\lambda g_{2}+\theta g_{3}\right\|=M / 2
$$


para todos $\lambda, \theta \in\{-1,1\}, \operatorname{logo}$

$$
\begin{aligned}
\left\|\mathrm{u}_{1}+\lambda \mathrm{u}_{2}+\theta \mathrm{u}_{3}\right\| & =\left\|T^{-1} g_{1}(k)+\lambda T^{-1} g_{2}(k)+\theta T^{-1} g_{3}(k)\right\| \\
& =\left\|T^{-1}\left(g_{1}+\lambda g_{2}+\theta g_{3}\right)(k)\right\| \\
& \leq M\left\|g_{1}+\lambda g_{2}+\theta g_{3}\right\|=M^{2} / 2
\end{aligned}
$$

Note que (5.10) contempla as desigualdades $\left(C_{1}\right)$ e $\left(C_{2}\right)$ da Definição 5.1 para $c=M^{2}$. Além disso, note que as desigualdades $\left(C_{1}\right)$ e $\left(C_{2}\right)$ são suficientes para a obtenção de (5.9) na prova do Lema 5.5, e então, seguindo procedimento análogo àquele, concluímos que

$$
\left\|\mathrm{u}_{i}-\mathrm{z}_{i} / 2\right\|^{2}+\left\|\mathrm{u}_{j}-\mathrm{z}_{j} / 2\right\|^{2} \leq\left(M^{4}-2\right) / 2, \forall i \neq j .
$$

Defina, para cada $1 \leq i \leq 3, t_{i}=\mathrm{u}_{i}-\mathrm{z}_{i} / 2$. Usando as três desigualdades acima concluímos que

$$
\left\|t_{1}\right\|^{2}+\left\|t_{2}\right\|^{2}+\left\|t_{3}\right\|^{2} \leq 3\left(M^{4}-2\right) / 4 .
$$

Pela regra do paralelogramo, segue que

$$
\sum_{\lambda, \theta \in\{-1,1\}}\left\|t_{1}+\lambda t_{2}+\theta t_{3}\right\|^{2}=4\left(\left\|t_{1}\right\|^{2}+\left\|t_{2}\right\|^{2}+\left\|t_{3}\right\|^{2}\right) \leq 3\left(M^{4}-2\right) .
$$

Então, fazendo uso de (5.3), inferimos que

$$
\sum_{\lambda, \theta \in\{-1,1\}}\left\|t_{1}+\lambda t_{2}+\theta t_{3}\right\| \leq 2 \sqrt{3} \sqrt{M^{4}-2} .
$$

Para cada par $\lambda, \theta \in\{-1,1\}$, temos por (5.11) que

$$
\begin{aligned}
\left\|\mathrm{z}_{1} / 2+\lambda \mathrm{z}_{2} / 2+\theta \mathrm{z}_{3} / 2\right\| & \leq\left\|\mathrm{u}_{1}+\lambda \mathrm{u}_{2}+\theta \mathrm{u}_{3}\right\|+\left\|t_{1}+\lambda t_{2}+\theta t_{3}\right\| \\
& \leq M^{2} / 2+\left\|t_{1}+\lambda t_{2}+\theta t_{3}\right\|,
\end{aligned}
$$

então

$$
\left\|\mathrm{z}_{1} / 2+\lambda \mathrm{z}_{2} / 2+\theta \mathrm{z}_{3} / 2\right\|^{2} \leq M^{4} / 4+M^{2}\left\|t_{1}+\lambda t_{2}+\theta t_{3}\right\|+\left\|t_{1}+\lambda t_{2}+\theta t_{3}\right\|^{2},
$$

e portanto, observando (5.12) e (5.13), obtemos

$$
\begin{aligned}
3 & =4\left(\left\|\mathrm{z}_{1} / 2\right\|^{2}+\left\|\mathrm{z}_{2} / 2\right\|^{2}+\left\|\mathrm{z}_{3} / 2\right\|^{2}\right) \\
& =\sum_{\lambda, \theta \in\{-1,1\}}\left\|\mathrm{z}_{1} / 2+\lambda \mathrm{z}_{2} / 2+\theta \mathrm{z}_{3} / 2\right\|^{2} \\
& \leq M^{4}+M^{2} \sum_{\lambda, \theta \in\{-1,1\}}\left\|t_{1}+\lambda t_{2}+\theta t_{3}\right\|+\sum_{\lambda, \theta \in\{-1,1\}}\left\|t_{1}+\lambda t_{2}+\theta t_{3}\right\|^{2} \\
& \leq M^{4}+3\left(M^{4}-2\right)+2 \sqrt{3} M^{2} \sqrt{M^{4}-2},
\end{aligned}
$$

então

$$
9 \leq 4 M^{4}+2 \sqrt{3} M^{2} \sqrt{M^{4}-2},
$$

contradizendo o fato de que $M^{2}$ é uma constante de Cambern.

A seguir provamos quatro lemas cujo propósito é descrever a influência dos v's sobre os w's nos conjuntos $\Gamma_{\mathrm{w}}(k, \mathrm{v})$. Ao fim do quarto lema teremos ferramentas suficientes para a prova da proposição final.

Lema 5.7. Sejam $U_{1}$ e $U_{2}$ conjuntos fechados de $S_{X}$ tais que $S_{X}=U_{1} \cup U_{2}$. Então um dos conjuntos $U_{1}$ e $U_{2}$ contém uma base ortonormal de $X$. 
Demonstração. Vamos provar esta proposição por indução na dimensão de $X$. Para $n=1$, é imediato. Suponha então que $n \geq 2$ e que o enunciado da proposição é verdadeiro quando a dimensão de $X$ é $n-1$. Como $S_{X}$ é a união dos fechados $U_{1}, U_{2}$ e é conexo, existe u $\in U_{1} \cap U_{2}$. Considere $Y=\mathrm{u}^{\perp}$. Então, a dimensão de $Y$ é $n-1 \mathrm{e}$

$$
S_{Y}=S_{X} \cap Y=\left(U_{1} \cap Y\right) \cup\left(U_{2} \cap Y\right) .
$$

Pela hipótese de indução, um dos conjuntos $U_{1} \cap Y$ e $U_{2} \cap Y$ contém uma base ortonormal $B$ de $Y$. Como $\mathrm{u} \in U_{1} \cap U_{2}$, segue que um dos conjuntos $U_{1}$ e $U_{2}$ contém o conjunto $B \cup\{\mathrm{u}\}$, que é uma base ortonormal de $X$.

Lema 5.8. Seja $k \in K$ e suponha que $\Phi(k)=\left\{s_{1}, s_{2}\right\}$ com $s_{1} \neq s_{2}$. Para todo $1 \leq i \leq 2$, faça

$$
V_{i}=\left\{\mathrm{v} \in S_{X}: s_{i} \in \Gamma_{\mathrm{w}}(k, \mathrm{v}) \text { para algum } \mathrm{w} \in X\right\} .
$$

Então $V_{1}$ ou $V_{2}$ tem uma base ortonormal de $X$.

Demonstração. Pela Proposição 5.4.(1) sabemos que $S_{X}=V_{1} \cup V_{2}$. Então, graças ao Lema 5.7 é suficiente mostrar que $V_{1}$ e $V_{2}$ são conjuntos fechados.

Por analogia, é suficiente provar que $V_{1}$ é um conjunto fechado. Seja $\left(\mathrm{v}_{j}\right)_{j \in \mathbb{N}}$ uma sequência em $V_{1}$ tal que $\mathrm{v}_{j} \rightarrow \mathrm{v}$. Pela definição de $V_{1}$, para cada $j \in \mathbb{N}$, fixe $\mathrm{w}_{j} \in X$ tal que

$$
s_{1} \in \Gamma_{\mathrm{w}_{j}}\left(k, \mathrm{v}_{j}\right) .
$$

Como a sequência $\left(\mathrm{w}_{j}\right)_{j \in \mathbb{N}}$ é limitada, ela admite uma subsequência convergente. Assim, podemos assumir que $\mathrm{w}_{j} \rightarrow \mathrm{w}$, para algum $\mathrm{w} \in X$. Dada $f \in C_{0}(K, X)$, por (5.14) deduzimos que

$$
\left\|T f\left(s_{1}\right)-\mathrm{w}_{j}\right\| \leq M \omega\left(k, f, \mathrm{v}_{j}\right), \forall j \in \mathbb{N},
$$

e portanto

$$
\left\|T f\left(s_{1}\right)-\mathrm{w}\right\| \leq M \omega(k, f, \mathrm{v}) .
$$

Isso prova que $s_{1} \in \Gamma_{\mathrm{w}}(k, \mathrm{v})$ e consequentemente $\mathrm{v} \in V_{1}$, o que completa a prova.

Lema 5.9. Sejam $k, k^{\prime} \in K$ com $k \neq k^{\prime} e \mathrm{v}^{\prime} \in X$ não-nulo. Se $\left\{\mathrm{v}_{1}, \ldots, \mathrm{v}_{n}\right\}$ é uma base ortogonal de $X$, então

$$
\bigcap_{i=1}^{n} \Gamma_{\mathrm{w}_{i}}\left(k, \mathrm{v}_{i}\right) \cap \Gamma_{\mathrm{w}^{\prime}}\left(k^{\prime}, \mathrm{v}^{\prime}\right)=\emptyset .
$$

Demonstração. Assuma que existe $s \in S$ satisfazendo

$$
s \in \Gamma_{\mathrm{w}_{i}}\left(k, \mathrm{v}_{i}\right) \cap \Gamma_{\mathrm{w}^{\prime}}\left(k^{\prime}, \mathrm{v}^{\prime}\right), \forall 1 \leq i \leq n,
$$

e veremos abaixo que $M^{2}$ não é uma constante de Cambern para $X$, contradizendo nossas hipóteses.

Devido à Proposição 5.4.3, podemos assumir que $\left\|\mathrm{v}_{i}\right\|=\left\|\mathrm{v}^{\prime}\right\|=M$, para todo $1 \leq i \leq n$. Como $\left\|\mathrm{w}^{\prime}\right\|=\left\|\mathrm{v}^{\prime}\right\| / M-L$ e $L=0$, segue que $\left\|\mathrm{w}^{\prime}\right\|=1$ e o mesmo raciocínio se aplica aos $\mathrm{w}_{i}$ 's, então

$$
\left\|\mathrm{w}_{i}\right\|=\left\|\mathrm{w}^{\prime}\right\|=1 \text { para todo } 1 \leq i \leq n .
$$

Como $k \neq k^{\prime}$, pelo Lema de Urysohn (veja a Seção 2.2) existem $f$ e $f^{\prime} \in C_{0}(K)$ satisfazendo:

(a) $f(K), f^{\prime}(K) \subset[0,1]$

(b) $f(k)=f^{\prime}\left(k^{\prime}\right)=1$;

(c) Os suportes de $f$ e $f^{\prime}$ são disjuntos.

Faça

$$
h^{\prime}=f^{\prime} \frac{\mathrm{v}^{\prime}}{2} \text { and } h_{i}=f \frac{\mathrm{v}_{i}}{2}, \forall 1 \leq i \leq n,
$$


$\mathrm{e}$

$$
\mathrm{u}^{\prime}=T h^{\prime}(s) \text { and } \mathrm{u}_{i}=T h_{i}(s), \forall 1 \leq i \leq n .
$$

Vejamos abaixo que $\mathrm{u}^{\prime}, \mathrm{u}_{1}, \ldots, \mathrm{u}_{n} \in X$ e $\mathrm{w}^{\prime}, \mathrm{w}_{1}, \ldots, \mathrm{w}_{n} \in S_{X}$ satisfazem simultaneamente as desigualdades da Definição 5.1 para $c=M^{2}$.

$\left(C_{1}\right)$ Dado $1 \leq i \leq n$, sabemos por (5.15) que $s \in \Gamma_{\mathrm{w}_{i}}\left(k, \mathrm{v}_{i}\right)$, o que, recordando a Definição 3.1 dos conjuntos de aproximação, nos dá

$$
\left\|T f(s)-\mathrm{w}_{i}\right\| \leq M \omega\left(k, f, \mathrm{v}_{i}\right), \quad \forall f \in C_{0}(K, X) .
$$

Por conta da propriedade (c), temos que $\left\|h^{\prime}+h_{i}\right\|=M / 2$. Além disso, pela propriedade (b),

$$
\left\|h^{\prime}(k)+h_{i}(k)-\mathrm{v}_{i}\right\|=\left\|0+\mathrm{v}_{i} / 2-\mathrm{v}_{i}\right\|=\left\|\mathrm{v}_{i}\right\| / 2=M / 2 .
$$

Consequentemente,

$$
\omega\left(k, h^{\prime}+h_{i}, \mathrm{v}_{i}\right)=\max \left\{\left\|h^{\prime}+h_{i}\right\|,\left\|h^{\prime}(k)+h_{i}(k)-\mathrm{v}_{i}\right\|\right\}=M / 2 .
$$

Portanto, de acordo com (5.16) e (5.17), inferimos que

$$
\begin{aligned}
\left\|\mathrm{u}^{\prime}+\left(\mathrm{u}_{i}-\mathrm{w}_{i}\right)\right\| & =\left\|\left(\mathrm{u}^{\prime}+\mathrm{u}_{i}\right)-\mathrm{w}_{i}\right\|=\left\|\left(T h^{\prime}(s)+T h_{i}(s)\right)-\mathrm{w}_{i}\right\| \\
& =\left\|T\left(h^{\prime}+h_{i}\right)(s)-\mathrm{w}_{i}\right\| \leq M \omega\left(k, h^{\prime}+h_{i}, \mathrm{v}_{i}\right)=\frac{M^{2}}{2}
\end{aligned}
$$

Por outro lado, aplicando a Proposição $5.4 .3 \operatorname{com} t=-1$ ao fato $s \in \Gamma_{\mathrm{w}_{i}}\left(k, \mathrm{v}_{i}\right)$, obtemos que $s \in \Gamma_{-\mathrm{w}_{i}}\left(k,-\mathrm{v}_{i}\right)$, e consequentemente

$$
\left\|T f(s)+\mathrm{w}_{i}\right\| \leq M \omega\left(k, f,-\mathrm{v}_{i}\right), \quad \forall f \in C_{0}(K, X) .
$$

Por um raciocínio análogo ao feito para (5.17), concluímos que

$$
\omega\left(k, h^{\prime}-h_{i},-\mathrm{v}_{i}\right)=M / 2 .
$$

E então de modo análogo à (5.18), obtemos

$$
\left\|\mathrm{u}^{\prime}-\left(\mathrm{u}_{i}-\mathrm{w}_{i}\right)\right\| \leq \frac{M^{2}}{2} .
$$

As desigualdades (5.18) e (5.19) correspondem às desigualdades $\left(C_{1}\right)$ da Definição 5.1 para $c=M^{2}$.

$\left(C_{2}\right)$ Dado $1 \leq i \leq n$, procedendo analogamente às provas de (5.16) e (5.17), concluimos que

$$
s \in \Gamma_{ \pm \mathrm{w}^{\prime}}\left(k^{\prime}, \pm \mathrm{v}^{\prime}\right) \text { e } \omega\left(k^{\prime}, h_{i} \pm h^{\prime}, \pm \mathrm{v}^{\prime}\right)=\frac{M}{2},
$$

e então que

$$
\left\|\mathrm{u}_{i} \pm\left(\mathrm{u}^{\prime}-\mathrm{w}^{\prime}\right)\right\|=\left\|T\left(h_{i} \pm h^{\prime}\right)(s) \mp \mathrm{w}^{\prime}\right\| \leq M \omega\left(k^{\prime}, h_{i} \pm h^{\prime}, \pm \mathrm{v}^{\prime}\right)=\frac{M^{2}}{2} .
$$

$\left(C_{3}\right)$ Dados $1 \leq i, j \leq n$, com $i \neq j$ e $\lambda, \theta \in\{-1,1\}$, fazendo uso de $(c)$ e do fato de que $\mathrm{v}_{i}$ e $\mathrm{v}_{j}$ são ortogonais, deduzimos que

$$
\begin{aligned}
\left\|\sqrt{2} h^{\prime}+\lambda h_{i}+\theta h_{j}\right\| & =\max \left\{\sqrt{2}\left\|h^{\prime}\right\|,\left\|\lambda h_{i}+\theta h_{j}\right\|\right\}=\max \left\{\sqrt{2} \frac{M}{2}, \frac{\left\|\lambda \mathrm{v}_{i}+\theta \mathrm{v}_{j}\right\|}{2}\right\} \\
& =\max \left\{\sqrt{2} \frac{M}{2}, \frac{M \sqrt{2}}{2}\right\}=\frac{M}{\sqrt{2}} .
\end{aligned}
$$


Consequentemente,

$$
\begin{aligned}
\left\|\sqrt{2} \mathrm{u}^{\prime}+\lambda \mathrm{u}_{i}+\theta \mathrm{u}_{j}\right\| & =\left\|\sqrt{2} T h^{\prime}(s)+\lambda T h_{i}(s)+\theta T h_{j}(s)\right\| \\
& =\left\|T\left(\sqrt{2} h^{\prime}+\lambda h_{i}+\theta h_{j}\right)(s)\right\| \\
& \leq M\left\|\sqrt{2} h^{\prime}+\lambda h_{i}+\theta h_{j}\right\|=\frac{M^{2}}{\sqrt{2}} .
\end{aligned}
$$

Lema 5.10. Seja $k \in K$ e suponha que $\Phi(k)$ não é um conjunto unitário. Então, para todo $s \in \Phi(k)$, $\Psi(s)$ não é um conjunto unitário.

Demonstração. Pela Proposição 5.4.(1), está claro que $\Phi(k) \neq \emptyset$. Logo, podemos fixar $s, s^{\prime} \in \Phi(k)$ com $s \neq s^{\prime}$. É simples checar que $k \in \Psi(s) \cap \Psi\left(s^{\prime}\right)$, bastando, para tal, fazer uso, em sequência, das propriedades (1) e (2) da Proposição 5.4.

Assuma por contradição que $\Psi(s)$ é um conjunto unitário. Então, é claro que $\Psi(s)=\{k\}$. Tome uma base ortogonal $\left\{\mathrm{v}_{1}, \ldots, \mathrm{v}_{n}\right\}$ de $X$. Pela propriedade (1) da Proposição 5.4 existem $\mathrm{w}_{1}, \ldots, \mathrm{w}_{n} \in X$ tais que

$$
k \in \Lambda_{\mathrm{w}_{i}}\left(s, \mathrm{v}_{i}\right), \forall 1 \leq i \leq n .
$$

Por outro lado, como $k \in \Psi\left(s^{\prime}\right)$ existem $\mathrm{v}^{\prime}, \mathrm{w}^{\prime} \in X$ tais que

$$
k \in \Lambda_{\mathrm{w}^{\prime}}\left(s^{\prime}, \mathrm{v}^{\prime}\right) .
$$

Portanto,

$$
k \in \bigcap_{i=1}^{n} \Lambda_{\mathrm{w}_{i}}\left(s, \mathrm{v}_{i}\right) \cap \Lambda_{\mathrm{w}^{\prime}}\left(s^{\prime}, \mathrm{v}^{\prime}\right),
$$

uma contradição com a versão simétrica da Proposição 5.9.

Finalmente provamos abaixo que os conjuntos $\Phi(k)$ e $\Psi(s)$ são unitários, concluindo a demonstração do Teorema 5.3.

Proposição 5.11. $\Phi(k)$ é um conjunto unitário para todo $k \in K$.

Demonstração. Sabemos, pela Proposição 5.4.(1), que $\Phi(k) \neq \emptyset$. Assuma que $\Phi(k)$ não é um conjunto unitário para algum $k \in K$. Pela Proposição 5.6 existem $s, s^{\prime} \in X$, com $s \neq s^{\prime}$, tais que $\Phi(k)=\left\{s, s^{\prime}\right\}$. De acordo com o Lema 5.8, podemos supor sem perda de generalidade que existe uma base ortogonal $\left\{\mathrm{v}_{1}, \ldots, \mathrm{v}_{n}\right\}$ de $X$ e $\mathrm{w}_{i} \in X, 1 \leq i \leq n$ tais que

$$
s \in \Gamma_{\mathrm{w}_{i}}\left(k, \mathrm{v}_{i}\right), \forall 1 \leq i \leq n .
$$

Por outro lado, pela Proposição 5.10 sabemos que $\Psi(s)$ não é um conjunto unitário. Assim, existe $k^{\prime} \in K$, com $k^{\prime} \neq k$, tal que $k^{\prime} \in \Psi(s)$. Além disso, pelo item (2) da Proposição 5.4 segue que existem $\mathrm{v}^{\prime}, \mathrm{w}^{\prime} \in X$ tais que

$$
s \in \Gamma_{\mathrm{w}^{\prime}}\left(k^{\prime}, \mathrm{v}^{\prime}\right) .
$$

Consequentemente,

$$
s \in \bigcap_{i=1}^{n} \Gamma_{\mathrm{w}_{i}}\left(k, \mathrm{v}_{i}\right) \cap \Gamma_{\mathrm{w}^{\prime}}\left(k^{\prime}, \mathrm{v}^{\prime}\right),
$$

uma contradição com a Proposição 5.9 . 



\section{Capítulo 6}

\section{Conclusões}

\subsection{Considerações Finais}

O estudo de generalizações do Teorema de Banach-Stone, historicamente, conta com uma extensa gama de técnicas, dentre as quais destacamos:

(a) técnicas com pontos extremais da bola unitária de $\left(C_{0}(K, X)\right)^{*}$.

(b) técnicas com propriedades de medida e o Teorema da Representação de Riesz.

As técnicas do tipo (b) foram as que possibilitaram os avanços mais importantes que estamos considerando nesse trabalho. Jarosz teve muitos de seus resultados como consequência do tipo de técnica (b). Quando enfim publicou [Jar89], foi a gênese de um novo tipo de técnica que culminou em nossa investigação atual.

É possível encontrar algumas conexões da técnica dos conjuntos de aproximação com as técnicas do tipo (a) e (b). O ponto de conexão mais importante está na demonstração do Lema 3.4, em que fazemos o uso das redes v-pico. A forma como utilizamos as redes v-pico em $k$ sugere conexões tanto com os operadores de valoração da bola unitária de $\left(C_{0}(K, X)\right)^{*}$ (tipo (a)), quanto com as medidas concentradas no ponto (tipo (b)).

A grande vantagem da técnica atual é que seus fundamentos não necessitam de entidades lineares. Em técnicas do tipo (a), por estarmos considerando o espaço dual de $C_{0}(K, X)$, pouco podemos derivar de relações entre os duais de $C_{0}(K, X)$ e $C_{0}(S, X)$ quando levamos em conta funções nãolineares. Já as técnicas do tipo (b) também dependem fortemente de que a função entre os espaços $C(K)$ seja linear, para assim induzir as medidas com as quais trabalham.

Sendo assim, temos a vantagem crucial de estarmos mais próximos do comportamento matemático do problema, e assim nos desvencilhamos das hipóteses necessárias para que se encaixem em teorias precedentes. Um grande indício da força da técnica está no fato de que, em todos os resultados provenientes da técnica dos conjuntos de aproximação, ou obtivemos uma versão não-linear que generaliza por completo a versão linear já conhecida, ou conseguimos melhorar a versão conhecida até então.

Talvez um defeito, que devamos considerar, seja ainda não ser possível definir os conjuntos de aproximação para espaços de Banach $X$ de dimensão infinita.

Certamente, os novos resultados, junto com a nova técnica, mudaram paradigmas inerentes ao problema e acrescentou resultados importantíssimos na linha. Isso sugere a continuidade da pesquisa em aplicações dos conjuntos de aproximação para obtenção de versões do Teorema de Banach-Stone nos demais contextos não analisados aqui.

\subsection{Sugestões para Pesquisas Futuras}

Uma possível direção para pesquisas futuras seria investigar generalizações do Teorema de Cambern para valores específicos de dimensões, a começar por $\mathbb{R}^{2}$. A definição de $\delta$ no Lema 5.2 implica numa dependência de $\delta$ com a dimensão $n$ de $X$. Além disso, o fato do Teorema de Cambern não 
poder ser estendido para o caso em que o espaço de Hilbert é dimensão infinita, indica que tal dependência de $\delta_{n}$ por $n$ deva satisfazer $\delta_{n} \rightarrow 0$ conforme $n \rightarrow 0$.

Problema 6.1. Seria possivel aumentar o $\delta_{2}$ dado pelo Teorema 1.24 quando $X=\mathbb{R}^{2}$ ? Em geral, seria possivel melhorar a dependência de $\delta_{n}$ pela dimensão $n$ de $X$ ?

É válido mencionar que para fazermos uso do Teorema 1.16, uma vez que a constante $M$ da quasi-isometria satisfaz $M \geq 1$, o espaço de Banach $X$ deve satisfazer $\mathcal{S}(X)>1$. Além disso, não temos nenhum resultado para isomorfismos satisfazendo $\|T\|\left\|T^{-1}\right\|>1$ quando o espaço de Banach $X$ tem $S(X)=1$. Tendo isso em vista, propomos o seguinte:

Problema 6.2. Suponha que $X$ é um espaço de Banach tal que existe $c>1$ satisfazendo a seguinte propriedade: para quaisquer espaços de Hausdorff localmente compactos $K$ e $S$ e uma $(M, L)$-quasiisometria bijetora $T$ de $C_{0}(K, X)$ sobre $C_{0}(S, X)$ com

$$
M^{2}<c,
$$

segue que $K$ e $S$ são homeomorfos.

Então,

(1) É verdade que $S(X)>1$ ?

(2) É verdade que $c \leq S(X)$ ?

(3) Segue que X é um espaço de dimensão finita?

Quando os espaços $K$ e $S$ são assumidos compactos enumeráveis, pelo Teorema de SierpinskiMazurkiewicz, temos que $K$ e $S$ são homeomorfos a certos espaços de ordinal $[0, \alpha]$ e $[0, \beta]$. Gordon [Gor70] provou que, quando $K$ e $S$ são espaços de ordinal, o Teorema de Banach-Stone é válido para isomorfismos $T$ satisfazendo $\|T\|\left\|T^{-1}\right\|<3$. Em [Ami65], Amir exibe contra-exemplos que atestam que a constante 3 do resultado de Gordon não pode ser aumentada. Nesse espírito, propomos o seguinte problema:

Problema 6.3. É possivel adicionar propriedades aos espaços $K$ e $S$ de modo a obter uma versão do Teorema de Banach-Stone que englobe $(M, L)$-quasi-isometrias entre $C_{0}(K)$ e $C_{0}(S)$ com $M \geq \sqrt{2}$ ?

Um tópico interessante dentro das versões lineares vetoriais do Teorema de Banach-Stone é determinar novos espaços que satisfazem a propriedade de Banach-Stone. Dizemos que um espaço de Banach $X$ satisfaz a propriedade de Banach-Stone se, para quaisquer espaços de Hausdorff localmente compactos $K$ e $S$, a existência de um isomorfismo isométrico de $C_{0}(K, X)$ sobre $C_{0}(S, X)$ implica que $K$ e $S$ são homeomorfos.

O Teorema 1.15 tem como consequência que todo espaço de Banach real $X \operatorname{com} \lambda(X)>1$ satisfaz a propriedade de Banach-Stone. Portanto, resta investigar quanto à tal propriedade para espaços de Banach reais com $\lambda(X)=1$.

Em adendo, conforme vimos na introdução, Sundaresan mostrou em [Sun73] que os espaços $\ell_{\infty}^{n}$ não satisfazem a propriedade de Banach-Stone. Vejamos o seguinte resultado obtido por Cambern [Cam77]:

Teorema 6.4. Seja $X$ um espaço de Banach real com dim $X=3$. Então $X$ satisfaz a propriedade de Banach-Stone se, e somente se, $X$ não é isometricamente isomorfo a $\ell_{\infty}^{3}$.

Tendo em vista o teorema acima, propomos o seguinte problema:

Problema 6.5. Quais espaços de Banach $X$ reais, de dimensão finita, com $\lambda(X)=1$, satisfazem a propriedade de Banach-Stone?

Por fim, um desafio interessante que resultaria em teoremas mais gerais, seria desenvolver uma versão da técnica para espaços de Banach $X$ de dimensão infinita. 


\section{Referências Bibliográficas}

[Ami65] D. Amir. On isomorphisms of continuous function spaces. Israel J. Math., 3:205-210, 1965. 1,42

[Ban33] S. Banach. Théorie des opérations linéaires. Monografje Matematyczne, Varsóvia, 1933. 1

[Beh78] E. Behrends. M-structure and the Banach-Stone theorem. Lecture Notes in Math. 736. Springer-Verlag, 1978. 1

[BL00] J. Benyamini e Y. Lindenstrauss. Geometric nonlinear functional analysis. Colloquium Publications 48. AMS, 2000. 2

[Bri08] M. R. Bridson. Geometric and combinatorial group theory. The Princeton Companion to Mathematics, Section IV.10, 2008. 2

[Cam66] M. Cambern. A generalized Banach-Stone theorem. Proc. Amer. Math. Soc., 17:396400, 1966. 1

[Cam67] M. Cambern. On isomorphisms with small bound. Proc. Amer. Math. Soc., 18:10621066, 1967. 1

[Cam76] M. Cambern. Isomorphisms of spaces of continuous vector-valued functions. Illinois J. Math., 20(1):1-11, 1976. 1, 5

[Cam77] M. Cambern. The Banach-Stone property and the weak Banach-Stone property in three-dimensional spaces. Proc. Amer. Math. Soc., 67(1):55-61, 1977. 42

[Cen73] B. Cengiz. A generalization of the banach-stone theorem. Proc. Amer. Math. Soc., 40(2):426-430, 1973. 7

[CGRV15] F. C. Cidral, E. M. Galego e M. A. Rincón-Villamizar. Optimal extensions of the Banach-Stone theorem. J. Math. Anal. Appl., 430(1):193-204, 2015. 1, 5

[Coh75] H. B. Cohen. A bound-two isomorphism between $C(X)$ Banach spaces. Proc. Amer. Math. Soc., 50(1):215-217, 1975. 1

[DK05] Y. Dutrieux e N. J. Kalton. Perturbations of isometries between $C(K)$-spaces. Studia Math., 166(2):181-197, 2005. 3

[GL90] J. Gao e K. S. Lau. On the geometry of spheres in normed linear spaces. J. Austral. Math. Soc. Ser. A, 48(1):101-112, 1990. 12

[GLZ14] G. Godefroy, G. Lancien e V. Zizler. The non-linear geometry of Banach spaces after Nigel Kalton. Rocky Mountain J. Math., 44(5):1529-1583, 2014. 2

[Gor70] Y. Gordon. On the distance coefficient between isomorphic function spaces. Israel J. Math., 8(4):391-397, 1970. 42 
[Gór11a] R Górak. Coarse version of the Banach-Stone theorem. J. Math. Anal. Appl., 377(1):406-413, 2011. 2, 3

[Gór11b] R. Górak. Perturbations of isometries between Banach spaces. Studia Math., 207(1):47$58,2011.3$

[GPdS16] E. M. Galego e A. L. Porto da Silva. An optimal nonlinear extension of Banach-Stone theorem. J. Funct. Anal., 271(8):2166-2176, 2016. 3

[GPdS17] E. M. Galego e A. L. Porto da Silva. A vector-valued Banach-Stone theorem with distortion $\sqrt{2}$. Pacific J. Math., 290(2):321-332, 2017. 6

[GPdS18a] E. M. Galego e A. L. Porto da Silva. An Amir-Cambern theorem for quasi-isometries of $c_{0}(k, x)$ spaces. Pacific J. Math., 297(1):87-100, 2018. 5

[GPdS18b] E. M. Galego e A. L. Porto da Silva. Quasi-isometries of $C_{0}(K, E)$ spaces which determine $K$ for all Euclidean spaces E. Studia Math., 243:233-242, 2018. 4

[GPdS19a] E. M. Galego e A. L. Porto da Silva. Isomorphisms of $C_{0}(K, X)$ spaces with large distortion. Math. Nachr., 292(5):996-1007, 2019. 6

[GPdS19b] E. M. Galego e A. L. Porto da Silva. Quasi-isometries on subsets of $c_{0}(k)$ and $c_{0}^{(1)}(k)$ spaces which determine $k$. Proc. Amer. Math. Soc., 147(8):3455-3470, 2019. 7

[GPdS19c] E. M. Galego e A. L. Porto da Silva. A solution to the cambern problem for finitedimensional hilbert spaces. Israel J. Math., 1:419-436, 2019. 7

[GPdS20] E. M. Galego e A. L. Porto da Silva. Nonlinear embeddings of spaces of continuous functions. Proc. Amer. Math. Soc., 148(4):1555-1566, 2020. 7

[Hol66] W. Holsztyński. Continuous mappings induced by isometries of spaces of continuous functions. Studia Math., 26(2):133-136, 1966. 7

[Jam64] R. C. James. Uniformly non-square Banach spaces. Ann. of Math., 80:542-550, 1964. 12

[Jar89] K. Jarosz. Nonlinear generalizations of the Banach-Stone theorem. Studia Math., 93(2):97-107, 1989. 2, 4, 41

[Jer50] M. Jerison. The space of bounded maps into a Banach space. Ann. Math., 52(2):309327, 1950. 1

[Kal08] N. J. Kalton. The nonlinear geometry of Banach spaces. Rev. Mat. Complut., 21(1):760, 2008. 2

[Meg12] R. E. Megginson. An introduction to Banach space theory, volume 183. Springer Science \& Business Media, 2012. 9, 10, 11

[Rud87] W. Rudin. Real and Complex Analysis. McGraw-Hill, 1987. 11, 31

[Sch76] J. J. Schäffer. Geometry of spheres in normed spaces. Marcel Dekker, New York, 1976. 12

[Sto37] M. H. Stone. Applications of the theory of Boolean rings to general topology. Trans. Amer. Math. Soc., 41(3):375-481, 1937. 1

[Sun73] K. Sundaresan. Spaces of continuous functions into a Banach space. Studia Math., 48:15-22, 1973. 5, 42

[Ves04] I. Vestfrid. Affine properties and injectivity of quasi-isometries. Israel J. Math., 141(1):185-210, 2004. 4 